\title{
Counting and construction of holomorphic primary fields in free CFT4 from rings of functions on Calabi-Yau orbifolds
}

\author{
Robert de Mello Koch, ${ }^{a}$ Phumudzo Rabambi, ${ }^{a}$ Randle Rabe ${ }^{a}$ \\ and Sanjaye Ramgoolam ${ }^{a, b}$ \\ ${ }^{a}$ National Institute for Theoretical Physics, \\ School of Physics and Mandelstam Institute for Theoretical Physics, \\ University of Witwatersrand, Wits, 2050, South Africa \\ ${ }^{b}$ Centre for Research in String Theory, School of Physics and Astronomy, \\ Queen Mary University of London, Mile End Road, London E1 4NS, U.K. \\ E-mail: robert@neo.phys.wits.ac.za, \\ Phumudzo.Rabambi@students.wits.ac.za, 1296569@students.wits.ac.za, \\ s.ramgoolam@qmul.ac.uk
}

ABSTRACT: Counting formulae for general primary fields in free four dimensional conformal field theories of scalars, vectors and matrices are derived. These are specialised to count primaries which obey extremality conditions defined in terms of the dimensions and left or right spins (i.e. in terms of relations between the charges under the Cartan subgroup of $\mathrm{SO}(4,2)$ ). The construction of primary fields for scalar field theory is mapped to a problem of determining multi-variable polynomials subject to a system of symmetry and differential constraints. For the extremal primaries, we give a construction in terms of holomorphic polynomial functions on permutation orbifolds, which are shown to be Calabi-Yau spaces.

KEYwords: AdS-CFT Correspondence, Conformal Field Theory, Space-Time Symmetries ArXIV EPRINT: 1705.06702 


\section{Contents}

1 Introduction 1

2 Representations of $s o(4,2)$ on multi-variable polynomials 4

3 Counting with so $(4,2)$ characters $\quad 9$

3.1 General counting formula 9

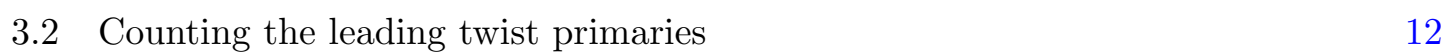

$\begin{array}{lll}3.3 & \text { Extremal primaries } & 14\end{array}$

4 Construction and construction with symmetric groups $\quad 17$

$\begin{array}{ll}4.1 & \text { Leading twist primaries } \\ & 18\end{array}$

4.2 Extremal primaries 22

$\begin{array}{lll}4.3 & \text { Palindromy properties } & 28\end{array}$

4.4 Gorenstein, Calabi-Yau and top-forms 29

5 Vector model primaries: symmetry breaking $S_{2 n} \rightarrow S_{n}\left[S_{2}\right] \quad 30$

6 Matrix model primaries $\quad 34$

$\begin{array}{lll}7 & \text { Summary and outlook } & 40\end{array}$

A Decomposing $\operatorname{Sym}^{n}\left(V_{+}\right)$for small values of $n \quad 41$

B Generating function of characters $\quad 42$

C The Hilbert series for $Z_{3}(s, x, y) \quad 43$

\section{Introduction}

In [1] we showed that free scalar four dimensional conformal field theory can be formulated as an infinite dimensional associative algebra, admitting a decomposition into linear representations of $\mathrm{SO}(4,2)$, and equipped with a bilinear product satisfying a non-degeneracy condition. This algebraic structure gives a formulation of the CFT4 as a two dimensional topological field theory (TFT2) with $\mathrm{SO}(4,2)$ invariance, where crossing symmetry is expressed as associativity of the algebra. TFT2 structure had previously been identified as a unifying structure in the study of combinatorics and correlators in BPS sectors of $N=4$ SYM, quiver gauge theories, matrix models, tensor models, and in Feynman graph combinatorics [2-5]. The theme of TFT2 as a powerful unifying structure for QFT combinatorics was also developed in [1] in the context of counting primary fields. In this paper we return 
to a systematic study of primaries in free field theories in four dimensions. We consider scalar, vector and matrix models. Another motivation for the detailed construction of primary fields four dimensional scalar QFT is that free field calculations have been found to be useful in calculating the anomalous dimensions of operators at the Wilson-Fischer fixed point in the epsilon expansion [6-12].

We start by developing some explicit formulae for the counting of primary fields, using characters of representations of $s o(4,2)$. This makes extensive use of previous literature on the subject, notably [13]. This is followed by considering the problem of constructing the primary fields. A useful remark is that the algebraic problem of finding composite fields of the form

$$
(\partial \cdots \partial \phi)(\partial \cdots \partial \phi) \cdots(\partial \cdots \partial \phi)
$$

where there are $n \phi$ fields involved, can be conveniently rephrased in terms of a question about multi-variable polynomial functions of $4 n$ variables: $\Psi\left(x_{\mu}^{I}\right)$ where $\mu$ runs over the space-time coordinates and $I$ runs from 1 to $n$. This relies on a function space realisation of the conformal algebra. We explain how this function space realisation arises naturally in radial quantization. The question of constructing primaries, when phrased in terms of the functions $\Psi\left(x_{\mu}^{I}\right)$ can be viewed as a many-body quantum mechanics problem, where $\Psi$ is a many-body wavefunction of $n$ particles moving on $\mathbb{R}^{4}$. These many-body wavefunctions have to obey three simple conditions:

- They have to obey Laplace's equation in each of the variables $x_{\mu}^{I}$ for $I=1 \cdots n$.

- They have to be invariant under the translation $x_{\mu}^{I} \rightarrow x_{\mu}^{I}+a_{\mu}$, for $\mu=1 \cdots 4$.

- They have to be invariant under permutations $x_{\mu}^{I} \rightarrow x_{\mu}^{\sigma(I)}$ for any permutation $\sigma \in S_{n}$.

An infinite class of solutions of the Laplacian condition are obtained by choosing a complex structure to identify $\mathbb{R}^{4}=\mathbb{C}^{2}$ so that $x_{\mu} \rightarrow(z, w, \bar{z}, \bar{w})$ and considering holomorphic functions of $z, w$. These primaries correspond to holomorphic polynomial functions on

$$
\left(\mathbb{C}^{2}\right)^{n} /\left(\mathbb{C}^{2} \times S_{n}\right)
$$

which can also be written as

$$
\left(\mathbb{C}^{n} / \mathbb{C} \times \mathbb{C}^{n} / \mathbb{C}\right) / S_{n}
$$

The modding out by $\mathbb{C}^{2}$ is the condition of invariance under the shift while the $S_{n}$ invariance comes from the permutation symmetry. A special class of these primary fields correspond to functions of $z$ only i.e functions on

$$
\left(\mathbb{C}^{n}\right) /\left(\mathbb{C} \times S_{n}\right)
$$

These primaries were constructed in [15] using an oscillator realization of the conformal algebra, which is close to the differential realization used here. An extensive study of the 
representations of $s o(4,2)$ on function spaces with emphasis on relations to quarternions, is developed in [16].

The association of primaries to functions on the orbifold has several interesting consequences. Since the holomorphic polynomial functions form a ring, and a class of primaries are in 1-1 correspondence with these functions, we are finding on a ring structure on this subspace of primary operators. This ring structure is different from the algebra structure related to the operator product expansion. The interplay between this product and the OPE would be an interesting subject for future study. The Hilbert series of the polynomial ring (1.2) has a very interesting palindromy property which we prove. The proof relies on an interesting algebraic structure based on symmetric groups in the problem. For fixed number of primaries $n$, this is

$$
\bigoplus_{k, l=0}^{\infty} \mathbb{C}\left(S_{n}\right) \otimes \mathbb{C}\left(S_{n}\right) \otimes \mathbb{C}\left(S_{k}\right) \otimes \mathbb{C}\left(S_{l}\right)
$$

where $\mathbb{C}\left(S_{n}\right)$ is the group algebra of the symmetric group $S_{n}$. As recently discussed in the context of Hilbert series for moduli spaces of supersymmetric vacua of gauge theories [17, 18], the palindromy property of Hilbert series is indicative that the ring being enumerated is Calabi-Yau. The precise mathematical statement is due to Stanley [19]. We show that the orbifold (1.2) indeed admits a unique non-singular nowhere-vanishing top-dimensional holomorphic form, which is inherited from the covering space.

Our work involves an interesting interplay between representations of $s o(4,2)$ and representations of symmetric groups. Let $V_{+}$be the lowest weight representation corresponding to local operators build from derivatives acting on the field $\phi$. The construction of primaries built from derivatives acting on $n$ copies of $\phi$, amounts to finding explicit formulae for the lowest weight states of irreducible representations in the symmetrized tensor product $\operatorname{Sym}^{n}\left(V_{+}\right)$. If we consider the primaries which arise at dimension $n+k$, of the class associated to the geometry (1.4) this can be mapped to a problem about multiplicities of $S_{n} \times S_{k}$ irreps in $V_{H}^{\otimes k}$ where $V_{H}$ is the $n-1$ dimensional representation of $S_{n}$. A formula for these multiplicities, derived in [15], is found to be useful in the study of the geometry of (1.2). The connection between representation theory of symmetric groups and that of non-compact groups has also been discussed in [20] in the context of higher spin theories.

We extend this approach to primary fields to the case of vector fields in four dimensions. The underlying orbifold geometry for holomorphic primary fields in this case is

$$
\left(\mathbb{C}^{2 n} / \mathbb{C} \times \mathbb{C}^{2 n} / \mathbb{C}\right) / S_{n}\left[S_{2}\right]
$$

The group $S_{n}\left[S_{2}\right]$ is the subgroup of $S_{2 n}$ which is generated by the $n$ pairwise permutations $(1,2),(3,4), \cdots,(n-1, n)$ along with the $n$ ! permutations of these pairs. It is called the wreath product of $S_{n}$ with $S_{2}$. We establish the palindromy property of the Hilbert series in this case. For the case of primary fields in the free theory of matrices in four dimensions, we again find the underlying orbifold geometry

$$
\left(\left(\mathbb{C}^{2}\right)^{n} \times S_{n}\right) /\left(\mathbb{C}^{2} \times S_{n}\right)=\left(\mathbb{C}^{n} / \mathbb{C} \times \mathbb{C}^{n} / \mathbb{C} \times S_{n}\right) / S_{n}
$$

with a palindromic Hilbert series. 
The paper is organized as follows. In section 2 we describe a realisation of the conformal algebra so $(4,2)$ in terms of differential operators acting on polynomial functions of spacetime coordinates $x_{\mu}$ in $\mathbb{R}^{4}$. This is related, by a duality which we explain, to the standard realization of the conformal algebra in terms of derivatives acting on a scalar field. In section 3 we obtain a number of useful general formulae for the counting of primary fields. The first step is to start from the character of the irrep $V_{+}$of $s o(4,2)$ which contains all the local operators consisting of derivatives acting on a single scalar field. This is a function of variables $s, x, y$ which keep track of dimension, left spin and right spin i.e eigenvalues of $D$ (the scaling operator) and $J_{L}, J_{R}$ (the Cartan generators for the two SU(2)'s in $\mathrm{SO}(4)=\mathrm{SU}(2) \times \mathrm{SU}(2))$. We then derive a generating function for the characters of all the symmetrised tensor products $\operatorname{Sym}^{n}\left(V_{+}\right)$. This is an application of the Cauchy identity. We describe a specialisation of these formulae relevant to what we call extremal primaries. These include the leading twist primaries studied in the context of deep inelastic scattering in QCD. Taylor expansion of the generating function leads to explicit results for $n=3,4$ which take the form of rational functions of $s, x, y$. In section 4 we describe the construction of the primary fields using the polynomial representations. A new counting formula for the extremal primaries is obtained by exploiting the permutation group algebras $\mathbb{C}\left(S_{n}\right) \times \mathbb{C}\left(S_{n}\right) \otimes \mathbb{C}\left(S_{k}\right) \otimes \mathbb{C}\left(S_{l}\right)$ in the problem of building primaries from $n$ fields $\phi$ and corresponding to polynomials of degree $k$ in one holomorphic variable and degree $l$ in the other. This is shown to be consistent with the derivation based on the Taylor expansion method of the previous section. These primary fields form a ring and the counting is recognised as a Hilbert series, which encodes aspects of the generators and relations of the ring. This is a ring of functions of an orbifold which we identify. The counting formula based on $S_{n} \times S_{k} \times S_{l}$ symmetry for the extremal sector is shown to have a palindromy property indicative of a Calabi-Yau nature of the orbifold. As further support for the Calabi-Yau nature of the orbifold, we construct the explicit top-dimensional holomorphic form. In section 5 we extend the results on counting and construction of primaries, and the underlying Calabi-Yau orbifold geometries, to the case of a four dimensional vector model. In section 6 we develop the story for the case of free four dimensional 1-matrix theory.

This paper is the extended version of an accompanying short paper [21].

\section{Representations of $s o(4,2)$ on multi-variable polynomials}

The generators of $\mathrm{SO}(4,2)$ form the algebra

$$
\begin{aligned}
{\left[K_{\mu}, P_{\nu}\right] } & =2 M_{\mu \nu}-2 D \delta_{\mu \nu} \\
{\left[D, P_{\mu}\right] } & =P_{\mu} \\
{\left[D, K_{\mu}\right] } & =-K_{\mu} \\
{\left[M_{\mu \nu}, K_{\alpha}\right] } & =\delta_{\nu \alpha} K_{\mu}-\delta_{\mu \alpha} K_{\nu} \\
{\left[M_{\mu \nu}, K_{\alpha}\right] } & =\delta_{\nu \alpha} P_{\mu}-\delta_{\mu \alpha} P_{\nu}
\end{aligned}
$$

The representations of this algebra play a central role when the constraints that conformal invariance places on the dynamics of a CFT are developed. To develop the representation 
theory, one uses the fact that there is a unique primary operator $\mathcal{O}$ for each irrep, formed by taking products of the fundamental fields of the theory and derivatives of these fields, with each other. The primary operator is distinguished because its dimension can not be lowered. Consequently, primaries are annihilated by the generator of special conformal transformations

$$
\left[K_{\mu}, \mathcal{O}\right]=0
$$

The complete irrep is then formed by acting on the primary $\mathcal{O}$ with traceless symmetric polynomials in the momenta $P_{\mu}$. The spectrum of dimensions of the primaries and their OPE coefficients provide a list of data that completely determines the correlation functions of local operators. Clearly then, it is interesting to determine the spectrum of primary operators of a conformal field theory. Our goal is to determine this list for the free bosonic field $\phi$ in four dimensions. The states corresponding to $\phi$ and its derivatives in the operatorstate correspondence consists of a lowest weight state $\left|v_{+}\right\rangle$

$$
\begin{aligned}
D \mid v_{+}> & =\mid v_{+}> \\
K_{\alpha} \mid v_{+}> & =0
\end{aligned}
$$

This state obeys

$$
K_{\alpha}\left(P_{\mu} P_{\mu} \mid 0>\right)=0
$$

which means that $P_{\mu} P_{\mu} \mid 0>$ can be set to zero to give an irreducible representation. The states in this representation are of the form

$$
\left(S^{(l)}\right)_{\mu_{1}, \mu_{2}, \cdots, \mu_{l}}^{\nu_{1}, \nu_{2}, \cdots, \nu_{l}} P_{\mu_{1}} P_{\mu_{2}} \cdots P_{\mu_{l}} \mid v^{+}>
$$

where $\left(S^{(l)}\right)_{\mu_{1}, \cdots, \mu_{l}}^{\nu_{1}, \cdots, \nu_{l}}$ is symmetric and traceless in both upper and lower indices.

Solving for the primaries $\mathcal{O}$ is a representation theory problem of finding the decomposition of the symmetrized tensor product $\operatorname{Sym}^{n}\left(V_{+}\right)$into irreducible representations. A particularly convenient realization of $V_{+}$is in terms of harmonic polynomials. Indeed polynomials of the form

$$
\left(S^{(l)}\right)_{\mu_{1}, \mu_{2}, \cdots, \mu_{l}}^{\nu_{1}, \nu_{2}, \cdots, \nu_{l}} x_{\mu_{1}} \cdots x_{\mu_{l}}
$$

are annihilated by the Laplacian

$$
\frac{\partial}{\partial x_{\alpha}} \frac{\partial}{\partial x_{\alpha}}
$$

hence are harmonic. The algebra $s o(4,2)$ is realised on these polynomials as $[1]$

$$
\begin{aligned}
K_{\mu} & =\frac{\partial}{\partial x_{\mu}} \\
P_{\mu} & =\left(x^{2} \partial_{\mu}-2 x_{\mu} x \cdot \partial-2 x_{\mu}\right) \\
D & =(x \cdot \partial+1) \\
M_{\mu \nu} & =x_{\mu} \partial_{\nu}-x_{\nu} \partial_{\mu}
\end{aligned}
$$


Thinking of $x_{\mu}$ as the co-ordinate of a particle, this is a single particle representation. The tensor product $V_{+}^{\otimes n}$ can be realized on a many-particle space of functions $\Psi\left(x_{\mu}^{I}\right)$, where $1 \leq I \leq n$ labels the particle number. The generators of $s o(4,2)$ now include

$$
\begin{aligned}
K_{\mu} & =\sum_{I=1}^{n} \frac{\partial}{\partial x_{\mu}^{I}} \\
P_{\mu} & =\sum_{I=1}^{n}\left(x^{I \rho} x_{\rho}^{I} \frac{\partial}{\partial x_{\mu}^{I}}-2 x_{\mu}^{I} x_{\rho}^{I} \frac{\partial}{\partial x_{\rho}^{I}}-2 x_{\mu}^{I}\right)
\end{aligned}
$$

along with the many-particle versions of $D, M_{\mu \nu}$ of (2.8). In this polynomial rep, the state of the scalar field $\lim _{|x| \rightarrow 0} \phi(x) \mid 0>$ corresponds to the harmonic function 1 .

This polynomial representation is naturally understood in the context of radial quantization. Towards this end, consider the mode expansion of the field

$$
\phi\left(x_{\mu}\right)=\sum_{l=0}^{\infty} \sum_{m \in V_{l}} a_{l ; m}^{\dagger} Y_{l, m}(x)+\sum_{l=0}^{\infty} \sum_{m \in V_{l}} a_{l ; m}|x|^{-2} Y_{l, m}\left(x^{\prime}\right)
$$

The sum over $m$ is over the states of the symmetric traceless tensor irrep $V_{l}$ of $\mathrm{SO}(4)$. Acting on the vacuum, which is annihilated by the $a_{l ; m}$ 's but not the $a_{l ; m}^{\dagger}$ 's, we have the usual operator-state correspondence. For example, we find

$$
\begin{aligned}
\lim _{x \rightarrow 0} \phi(x)|0\rangle & =a_{0 ; 0}^{\dagger}|0\rangle \equiv|\phi\rangle \\
\lim _{x \rightarrow 0} \partial_{\mu} \phi(x)|0\rangle & =a_{1 ; \mu}^{\dagger}|0\rangle \equiv\left|\partial_{\mu} \phi\right\rangle \\
\lim _{x \rightarrow 0} \partial_{\mu} \partial_{\mu} \phi(x)|0\rangle & =0
\end{aligned}
$$

The last equation above is expected because the free scalar field is a representation with null states. It expresses the free equation of motion. The scalar field and all its derivatives as $x \rightarrow 0$ lead to states in an irreducible lowest weight representation $V$ of $\mathrm{SO}(4,2)$, consisting of a lowest weight state of dimension $\Delta=1$ along with states with higher dimension.

Let us rewrite the positive part of the radial mode expansion

$$
\phi^{+}(x)|0\rangle=\sum_{l=0}^{\infty} a_{l ; \mu_{1}, \cdots, \mu_{l}}^{\dagger}\left(S^{(l)}\right)_{\nu_{1}, \cdots, \nu_{l}}^{\mu_{1}, \cdots, \mu_{l}} x^{\nu_{1}} \cdots x^{\nu_{l}}|0\rangle
$$

where $S^{(l)}$ is a projector, projecting to symmetric traceless tensors. We take $a_{l ; \mu_{1} \cdots \mu_{l}}^{\dagger}$ to be symmetric and traceless in the $\mu$ indices. $S^{(l)}$ is symmetric and traceless in the $\mu$ as well as the $\nu$ indices. The operator state map identifies

$$
\lim _{x \rightarrow 0} \partial_{\mu_{1}} \cdots \partial_{\mu_{l}} \phi(x)|0\rangle=\left(S^{(l)}\right)_{\mu_{1}, \cdots, \mu_{l}}^{\nu_{1}, \cdots, \nu_{l}} a_{l ; \nu_{1} \cdots \nu_{l}}^{\dagger}|0\rangle
$$

Note that we have a duality

$$
\begin{aligned}
\left.\left(\left(S^{(l)}\right)_{\mu_{1}, \cdots, \mu_{l}}^{\nu_{1}, \cdots, \nu_{l}} a_{l ; \nu_{1} \cdots \nu_{l}}^{\dagger}|0\rangle\right)^{\dagger}, \phi(x)|0\rangle\right) & =\left\langle 0\left|a_{l ; \nu_{1} \cdots \nu_{l}}\left(S^{(l)}\right)_{\mu_{1}, \ldots, \mu_{l}}^{\nu_{1}, \cdots, \nu_{l}} \phi(x)\right| 0\right\rangle \\
& =\left(S^{(l)}\right)_{\mu_{1}, \cdots, \mu_{l}}^{\alpha_{1}, \cdots, \alpha_{l}} x_{\alpha_{1}} \cdots x_{\alpha_{l}}
\end{aligned}
$$


where we have used the projector property of $S^{(l)}$. Unpacking this a little, if we apply $\partial_{\mu}$ to the local operator, go to zero to get the corresponding state and then do the duality, we will get a new polynomial as the outcome

$$
\lim _{x \rightarrow 0} \partial_{\mu} \partial_{\mu_{1}} \cdots \partial_{\mu_{l}} \phi(x)|0\rangle=\left(S^{(l)}\right)_{\mu, \mu_{1}, \cdots, \mu_{l}}^{\nu, \nu_{1}, \cdots, \nu_{l}} a_{l+1 ; \nu, \nu_{1} \cdots \nu_{l}}^{\dagger}|0\rangle
$$

If we take the overlap of this with $\phi(x)|0\rangle$ then we get

$$
\left(S^{(l)}\right)_{\mu, \mu_{1}, \cdots, \mu_{l}}^{\nu, \nu_{1}, \cdots, \nu_{l}} x_{\nu} x_{\nu_{1}} \cdots x_{\nu_{l}}
$$

This polynomial of degree one higher is related to the previous polynomial by applying $P_{\mu}=\left(x^{2} \partial_{\mu}-2 x_{\mu}(x \cdot \partial+1)\right)$. We have the following identifications between operators and states, and then states and polynomials

$$
\begin{aligned}
\mathcal{O} & \rightarrow|\mathcal{O}\rangle \rightarrow P_{\mathcal{O}}(x) \\
\partial_{\mu} \mathcal{O} & \rightarrow\left|\partial_{\mu} \mathcal{O}\right\rangle \rightarrow P_{\mu} P_{\mathcal{O}}(x)
\end{aligned}
$$

This provides a concrete correspondence between applying $\partial_{\mu}$ to local operators made from a scalar, and applying $P_{\mu}$ as the dual differential operator on dual polynomials.

Primaries in the free theory are given by acting with traceless symmetric polynomials in momenta, on the scalar field. Tracelessness is often implemented $[22,23]$ by using variables $z \cdot x^{I}=z^{\mu} x_{\mu}^{I}$ with $z^{\mu}$ a null vector, i.e. $z^{\mu} z_{\mu}=0$. Thanks to the fact that $z^{\mu}$ is null, any polynomial in $z \cdot x^{I}$ automatically gives a traceless symmetric polynomial in $x_{\mu}^{I}$ after the $z^{\mu}$ s are stripped away. In what follows we will solve the algebraic primary problem, to obtain a polynomial that corresponds to the primary. To obtain the primary operator written in terms of the original scalar field, we need to translate between the polynomials and operators. For the current polynomials, the translation between polynomials and operators is

$$
(z \cdot \partial)^{k} \phi \leftrightarrow(-1)^{k} 2^{k} k !(z \cdot x)^{k}
$$

This construction is convenient because of its simplicity. However, it is not completely general, since there are primary operators that are not symmetric in their indices and hence can't be represented as a polynomial in $z \cdot x$. The general discussion makes use of projectors that project from symmetric tensors to traceless symmetric tensors. It is useful to consider a concrete example. The tensors of ranks 2 and 3 are given by

$$
\begin{aligned}
\left(S^{(2)}\right)_{\mu \nu}^{\alpha \beta} & =\delta_{\mu}^{\alpha} \delta_{\nu}^{\beta}-\frac{1}{4} \delta_{\mu \nu} \delta^{\alpha \beta} \\
\left(S^{(3)}\right)_{\mu \nu \rho}^{\alpha \beta \gamma} & =\delta_{\mu}^{\alpha} \delta_{\nu}^{\beta} \delta_{\rho}^{\gamma}-\frac{1}{6}\left(\delta_{\mu \nu} \delta^{\alpha \beta} \delta_{\rho}^{\gamma}+\delta_{\mu \rho} \delta^{\alpha \gamma} \delta_{\nu}^{\beta}+\delta_{\mu}^{\alpha} \delta^{\beta \gamma} \delta_{\nu \rho}\right)
\end{aligned}
$$

These operators are projectors in the Brauer algebra of tensor operators that commute with $\mathrm{SO}(4)[24]$

$$
\begin{aligned}
& S^{(2)}=1-\frac{C_{12}}{4} \\
& S^{(3)}=1-\frac{1}{6}\left(C_{12}+C_{13}+C_{23}\right)
\end{aligned}
$$


The terms correcting the 1 above subtract off the trace of the tensors they act on. They satisfy

$$
\left(S^{(n)}\right)^{2} P_{n}=S^{(n)} P_{n}
$$

where $P_{n}$ projects onto the totally symmetric polynomials of degree $n$

$$
P_{n}=\frac{1}{n !} \sum_{\sigma \in S_{n}} \sigma
$$

The multiplication (2.22) is in the Brauer algebra, where loops are assigned the value of 4. These elements of the Brauer algebra are completely determined by the projector property (2.22) and the property that they start with 1 . In general

$$
P_{\mu_{1}} \cdots P_{\mu_{k}} \cdot 1=(-1)^{k} 2^{k} k !\left(S^{(k)}\right)_{\mu_{1} \cdots \mu_{k}}^{\nu_{1} \cdots \nu_{k}} x_{\nu_{1}} \cdots x_{\nu_{k}}
$$

The above factor is easily obtained by deriving a recursion formula. Note that the term $x^{2} \partial_{\mu}$ does not raise the rank of the tensor. The other two terms both raise the rank by one, which then leads to the recursion relation. In the many-particle realization such a traceless polynomial made of the $I$ 'th coordinates corresponds to derivatives acting on the $I^{\prime}$ th copy of $\phi$ in a sequence of $n$ of these.

To construct primaries using $n$ scalar fields we consider a multi-particle system with $x_{\mu}^{I}$ the coordinates of the $n$ particles. Primaries at dimension $n+k$ are obtained by allowing $k$ derivatives to act on the $n$ fields. In the dual polynomial language, states at dimension $n+k$ in $V^{\otimes n}$ correspond to polynomials in $x_{\mu}^{I}$ of degree $k$. Primaries at dimension $n+k$ correspond to degree $k$ polynomials $\Psi\left(x_{\mu}^{I}\right)$ that obey the conditions

$$
\begin{aligned}
K_{\mu} \Psi & =\sum_{I} \frac{\partial}{\partial x_{\mu}^{I}} \Psi=0 \\
\mathcal{L}_{I} \Psi & =\sum_{\mu} \frac{\partial}{\partial x_{\mu}^{I}} \frac{\partial}{\partial x_{\mu}^{I}} \Psi=0 \\
\Psi\left(x_{\mu}^{I}\right) & =\Psi\left(x_{\mu}^{\sigma(I)}\right)
\end{aligned}
$$

The first condition above is the familiar condition that the special conformal generator annihilates primary operators. The second condition implements the free scalar equation of motion which implies that the image of states like $P_{\mu}^{I} P_{\mu}^{I}$, with only $\mu$ summed, in the Fock space, is zero. This null state appears because the dimension of free scalar field saturates a unitarity bound. To see that the second constraint is indeed implementing the equation of motion, note that with the second of (2.8) we can calculate

$$
P_{\mu} P_{\mu}=x^{4} \partial_{\mu} \partial_{\mu}
$$

Simplifying the product of differential operators, it is simple to verify that terms like $x^{2}$, $x^{2} x \cdot \partial$ and $x^{2} x_{\mu} x_{\nu} \partial_{\mu} \partial_{\nu}$ cancel out. The final condition in (2.25) above ensures that our polynomials are $S_{n}$ invariant. By constructing $S_{n}$ invariant polynomials, we are implementing the bosonic statistics of the scalar field. 
In what follows we will focus on primaries (and hence polynomials) that transform in a definite representation of the $\mathrm{SO}(4)=\mathrm{SU}(2) \times \mathrm{SU}(2)$ subgroup of $\mathrm{SO}(4,2)$. To make the $\mathrm{SO}(4)$ transformation properties of the polynomials more transparent, our construction makes use of the complex coordinates

$$
\begin{array}{ll}
z=x_{1}+i x_{2} & w=x_{3}+i x_{4} \\
\bar{z}=x_{1}-i x_{2} & \bar{w}=x_{3}-i x_{4}
\end{array}
$$

This amounts to choosing an isomorphism between $\mathbb{R}^{4}$ and $\mathbb{C}^{2}=\mathbb{C} \times \mathbb{C}$. In our conventions, these coordinates have the following $\left(j_{L}^{3}, j_{R}^{3}\right)$ charge assignments

$$
\begin{array}{ll}
z \leftrightarrow\left(\frac{1}{2}, \frac{1}{2}\right) & \bar{z} \leftrightarrow\left(-\frac{1}{2},-\frac{1}{2}\right) \\
w \leftrightarrow\left(\frac{1}{2},-\frac{1}{2}\right) & \bar{w} \leftrightarrow\left(-\frac{1}{2}, \frac{1}{2}\right)
\end{array}
$$

We will construct a class of primaries corresponding to holomorphic polynomial functions on the orbifold

$$
\left(\mathbb{C}^{2}\right)^{n} /\left(\mathbb{C}^{2} \times S_{n}\right)
$$

The division by $\mathbb{C}^{2}$ is a consequence of the first of (2.25). These will not form the complete set of primaries but a well-defined subspace of primaries, which we will call extremal. Before explaining this construction in more detail we show, in the next section, how characters of $s o(4,2)$ representations can be used to get a complete counting of general primaries built from $n$ fields. We will then specialize to the extremal primaries.

\section{Counting with $s o(4,2)$ characters}

In this section our goal is to enumerate the $\mathrm{SO}(4,2)$ irreducible representations appearing among the composite fields made out of $n=2,3, \cdots$ fundamental fields. These multiplicities will, for example, compute the spectrum of primary operators in the free $\mathrm{CFT}_{4}$. This enumeration entails decomposing, into irreducible representations, the symmetrized tensor product $\operatorname{Sym}^{n}\left(V_{+}\right)$, where $V_{+}=D_{[1,0,0]}$ in the notation of [13]. The three integer labels in $D_{\left[\Delta, j_{L}, j_{R}\right]}$ are the dimension and two $\mathrm{SO}(4)$ spins. After obtaining a general formula in terms of an infinite product, we specialize to primaries that obey extremality conditions, that relate their dimension to their spin. For these primaries using results from [15], we find simple explicit formulas for the counting.

\subsection{General counting formula}

Consider a matrix $M$ belonging to any matrix representation $R$ of $\mathrm{SO}(4,2)$. A key result for the analysis of this section is

$$
\frac{1}{\operatorname{det}(1-t M)}=\sum_{n=0}^{\infty} t^{n} \chi_{\operatorname{Sym}^{n}(R)}(M)
$$


This is a special case of the Cauchy identity which states that

$$
\prod_{i=1}^{N} \prod_{j=1}^{M} \frac{1}{\left(1-t x_{i} y_{j}\right)}=\sum_{n=0}^{\infty} \sum_{R \vdash n} \chi_{R}(x) \chi_{R}(y)
$$

where $\chi_{R}$ is a Schur polynomial in the $N$ variables $x_{i}$ and the $M$ variables $y_{j}$, labelled by a Young diagram $R$ with $n$ boxes and height no larger that the minimum of $M, N$. When one of these variables is 1 , then we sum over single-row Young diagrams. This formula (3.1) is also easily proved by using the identity (this is just a statement of Wick's theorem)

$$
\begin{aligned}
\left(I_{n}\right)_{i_{1} \cdots i_{n}}^{j_{1} \cdots j_{n}} & =\frac{1}{\pi^{N}} \int \prod_{i=1}^{N} d x_{i} d y_{i} e^{-\sum_{k} z_{k} \bar{z}^{k}} \frac{1}{n !} z_{i_{1}} \cdots z_{i_{n}} \bar{z}^{j_{1}} \cdots \bar{z}^{j_{n}} \\
& =\frac{1}{n !} \sum_{\sigma \in S_{n}} \delta_{i_{\sigma(1)}}^{j_{1}} \delta_{i_{\sigma(2)}}^{j_{2}} \cdots \delta_{i_{\sigma(n)}}^{j_{n}}
\end{aligned}
$$

to evaluate

$$
\frac{1}{\pi^{N}} \int \prod_{i=1}^{N} d x_{i} d y_{i} e^{-\sum_{i, j} z_{i}\left(\delta_{j}^{i}-t M_{j}^{i}\right) \bar{z}^{j}}=\frac{1}{\operatorname{det}(1-t M)}
$$

Now, apply (3.1) to the case that

$$
M=s^{D} x^{J_{3, L}} y^{J_{3, R}}
$$

and specialize to the representation $V_{+}$spanned by the free scalar and all the derivatives acting on it. Here we have chosen $D, J_{3, L}, J_{3, R}$ to span the Cartan subalgebra of $\mathrm{SO}(4,2)$. It is straight forward to see that

$$
\frac{1}{\operatorname{det}(1-t M)}=\prod_{q=0}^{\infty} \prod_{a=-\frac{q}{2}}^{\frac{q}{2}} \prod_{b=-\frac{q}{2}}^{\frac{q}{2}} \frac{1}{1-t s^{q+1} x^{a} y^{b}}
$$

This generating function of the characters of the symmetrized tensor products of the free scalar representation will be denoted by $\mathcal{Z}(t, s, x, y)$. So we have

$$
\mathcal{Z}(t, s, x, y)=\prod_{q=0}^{\infty} \prod_{a=-\frac{q}{2}}^{\frac{q}{2}} \prod_{b=-\frac{q}{2}}^{\frac{q}{2}} \frac{1}{1-t s^{q+1} x^{a} y^{b}}=\sum_{n=0}^{\infty} t^{n} \chi_{\operatorname{Sym}^{n}\left(V_{+}\right)}(s, x, y)
$$

where we have denoted $\chi_{\mathrm{Sym}^{n}\left(V_{+}\right)}(M)$ by $\chi_{\mathrm{Sym}^{n}\left(V_{+}\right)}(s, x, y)$. The characters for $\operatorname{Sym}^{n}\left(V_{+}\right)$ follow by developing the infinite product above in a Taylor series in $t$. The decomposition of $\operatorname{Sym}^{n}\left(V_{+}\right)$into irreps is now achieved by writing $\chi_{\operatorname{Sym}^{n}\left(V_{+}\right)}(s, x, y)$ as a sum of characters $\chi_{\left[\Delta, j_{1}, j_{2}\right]}(s, x, y)$ of $M$, in the irrep of dimension $\Delta$ and spins $j_{1}, j_{2}$

$$
\chi_{\operatorname{Sym}^{n}\left(V_{+}\right)}(s, x, y)=\sum_{\Delta, j_{1}, j_{2}} N_{\left[\Delta, j_{1}, j_{2}\right]} \chi_{\left[\Delta, j_{1}, j_{2}\right]}(s, x, y)
$$

The coefficients $N_{\left[\Delta, j_{1}, j_{2}\right]}$ are non-negative integers, counting the number of times irrep $\mathcal{A}_{\left[\Delta, j_{1}, j_{2}\right]}$ (in the notation of [13]) appears in $\operatorname{Sym}^{n}\left(V_{+}\right)$). If we restrict to the case that 
$n \geq 3$, the only characters $\chi_{\left[\Delta, j_{1}, j_{2}\right]}(s, x, y)$ which contribute are labeled by dimensions $\Delta$ that do not saturate the unitarity bound and hence do not have any null states. In this case we have [13]

$$
\chi_{\left[\Delta, j_{1}, j_{2}\right]}(s, x, y)=\frac{s^{\Delta} \chi_{j_{1}}(x) \chi_{j_{2}}(y)}{(1-s \sqrt{x y})\left(1-s \sqrt{\frac{x}{y}}\right)\left(1-s \sqrt{\frac{y}{x}}\right)\left(1-\frac{s}{\sqrt{x y}}\right)}
$$

It is useful to define

$$
Z_{n}(s, x, y) \equiv \sum_{\Delta, j_{1}, j_{2}} N_{\left[\Delta, j_{1}, j_{2}\right]} s^{\Delta} \chi_{j_{1}}(x) \chi_{j_{2}}(y)
$$

It follows that

$$
Z_{n}(s, x, y)=(1-s \sqrt{x y})\left(1-s \sqrt{\frac{x}{y}}\right)\left(1-s \sqrt{\frac{y}{x}}\right)\left(1-\frac{s}{\sqrt{x y}}\right) \chi_{\operatorname{Sym}^{n}(V)}(s, x, y)
$$

The right hand side of this last equation is precisely a sum of (products of) $\mathrm{SU}(2)$ characters, so we can treat this, following [14], using the orthogonality of SU(2) characters. The result is most easily stated in terms of the generating function

$$
G_{n}(s, x, y)=\sum_{d=0}^{\infty} \sum_{j_{1}, j_{2}} N_{\left[n+d, j_{1}, j_{2}\right]} s^{n+d} x^{j_{1}} y^{j_{2}}
$$

which is given by

$$
G_{n}(s, x, y)=\left[\left(1-\frac{1}{x}\right)\left(1-\frac{1}{y}\right) Z_{n}(s, x, y)\right]_{\geq}
$$

where the subscript $\geq$ is a notation to indicate that the above function should first be expanded as a Laurent series in both $x$ and $y$, and then negative powers of $x$ and $y$ should be discarded. The infinite product in the above formula makes it difficult to evaluate $G_{n}(s, x, y)$ in closed form. For that reason, in the next section, we focus on specific classes of primaries for which $G_{n}(s, x, y)$ can be evaluated.

To end this section let us explain how the above derivation is generalized when irreps that include null states appear in the tensor product $\operatorname{Sym}^{n}\left(V_{+}\right)$. This is the case when $n=2$. Naively computing $G_{2}(s, x, y)$ using (3.13), we obtain the following terms

$$
G_{2}(s, x, y)=s^{2}+s^{4} x y-s^{5} \sqrt{x} \sqrt{y}+s^{6} x^{2} y^{2}-s^{7} x^{3 / 2} y^{3 / 2}+\cdots
$$

The negative coefficients in the above expansion show this answer is manifestly wrong. The problem is that we have some null states that have not been removed correctly. There are two types of primaries that appear in the above sum. We have a primary with $\Delta=2$ and $j_{1}=j_{2}=0$ and primaries with $\Delta=2+2 j$ and $j_{1}=j_{2}=j$ for $j=1,2,3, \ldots$. The condition for a short multiplet [25] is that $\Delta=f\left(j_{1}\right)+f\left(j_{2}\right)$ with $f(j)=0$ if $j=0$ or $f(j)=j+1$ if $j>0$. The primary with $\Delta=2$ and $j_{1}=j_{2}=0$ is not short and nothing needs to be subtracted. The primaries with $\Delta=2+2 j$ and $j_{1}=j_{2}=j$ are short irreps and hence have 
null states. These null states (and their descendants) must be removed. To understand how this is done, note that the primary with $\Delta=2+2 j$ and $j_{1}=j_{2}=j$ is a conserved higher spin current $J^{\mu_{1} \mu_{2} \cdots \mu_{j}}$ and the null state is nothing but the conservation law

$$
\partial_{\mu} J^{\mu \mu_{2} \cdots \mu_{j}}=0
$$

The null state thus has $\Delta=3+2 j$ and $j_{1}=j_{2}=j-\frac{1}{2}$ and so the subtraction of null states is achieved by removing the primary that does not need to be subtracted, dividing by $1-s / \sqrt{x y}$ and then putting the original primary back in. In the end we have

$$
\begin{aligned}
G_{2}(s, x, y) & =\left[\left(1-\frac{1}{x}\right)\left(1-\frac{1}{y}\right)\left(Z_{2}(s, x, y)-s^{2}\right) \frac{1}{1-\frac{s}{\sqrt{x y}}}\right]_{\geq}+s^{2} \\
& =\sum_{j=0}^{\infty} s^{2+2 j} x^{j} y^{j}
\end{aligned}
$$

This is indeed the correct result.

\subsection{Counting the leading twist primaries}

Consider the leading twist primaries, which have quantum numbers $\left[\Delta, j_{1}, j_{2}\right]=\left[n+q, \frac{q}{2}, \frac{q}{2}\right]$. Each such primary operator comes in a complete spin multiplet of $(q+1)^{2}$ operators. Choosing the operator with highest spin corresponds to studying polynomials constructed using only the single complex variable $z$, as we can see from (2.28). This corresponds to the fact that all primaries are constructed using a single component $P_{z}$ of the momentum four vector operator. We will now count the leading twist primaries by counting this highest spin operator in each multiplet. Denote the corresponding generating function by $G_{n}^{\max }(s, x, y)$. To determine this generating function we will modify the above results in three ways:

1. We modify the formula (3.7) by replacing $\chi_{\operatorname{Sym}^{n}(V)}(s, x, y)$ with a new function $\chi_{n}^{\max }(s, x, y)$, and we keep only the highest spin state in the product

$$
\prod_{q=0}^{\infty} \frac{1}{1-t s^{q+1} x^{\frac{q}{2}} y^{\frac{q}{2}}}=\sum_{n=0}^{\infty} t^{n} \chi_{n}^{\max }(s, x, y)
$$

2. The leading twist primaries are all constructed using a single component of the momentum, that raises both the left and right spin maximally. Consequently in (3.13) we keep only the factor that corresponds to this component of the momentum, which amounts to replacing

$$
(1-s \sqrt{x y})\left(1-s \sqrt{\frac{x}{y}}\right)\left(1-s \sqrt{\frac{y}{x}}\right)\left(1-\frac{s}{\sqrt{x y}}\right) \rightarrow(1-s \sqrt{x y})
$$

3. For each spin multiplet we keep only 1 state so there is no longer any need to replace the multiplet of spin states by a single state when we count. Thus in (3.13) we replace

$$
\left(1-\frac{1}{x}\right)\left(1-\frac{1}{y}\right) \rightarrow 1
$$


The final result is

$$
G_{n}^{\max }(s, x, y)=\chi_{n}^{\max }(s, x, y)(1-s \sqrt{x y})
$$

In this formula we don't need to track the dependence on $x$ and $y$ since for this class of primaries, once $n$ and the dimension of the operator is specified, the spins are determined. For simplicity then, we will study

$$
\sum_{n=0}^{\infty} t^{n} G_{n}^{\max }(s)=\sum_{n=0}^{\infty} t^{n}(1-s) \chi_{n}^{\max }(s)=(1-s) \prod_{q=0}^{\infty} \frac{1}{1-t s^{q+1}}
$$

To extract $G_{n}^{\max }(s)$, we need to develop the infinite product above in a Taylor series in $t$. To do this we introduce the functions

$$
F(t, s)=\prod_{q=0}^{\infty} \frac{1}{1-t s^{q+1}} \quad \frac{\partial F}{\partial t}=f_{1} F \quad f_{k}=\frac{\partial^{k-1} f_{1}}{\partial t^{k-1}}
$$

It is straightforward to find $F(0, s)=1$ and

$$
f_{k}(t, s)=(k-1) ! \sum_{a=0}^{\infty} \frac{s^{k a+k}}{\left(1-t s^{a+1}\right)^{k}} \quad f_{k}(0, s)=(k-1) ! \frac{s^{k}}{1-s^{k}}
$$

Using these quantities, we have

$$
\frac{\partial^{n} F}{\partial t^{n}}=\sum_{n_{1}, \cdots, n_{q}} \sum_{k_{1}, \cdots, k_{q}} \frac{\left(n_{1} k_{1}+\cdots+n_{q} k_{q}\right) !}{n_{1} ! \cdots n_{q} !\left(k_{1} !\right)^{n_{1}} \cdots\left(k_{q} !\right)^{n_{q}}} f_{k_{1}}^{n_{1}} \cdots f_{k_{q}}^{n_{q}} \delta_{n, n_{1} k_{1}+\cdots n_{q} k_{q}} F
$$

Inserting the formulas for the $f_{k}$ 's we have

$$
\begin{aligned}
\left.\frac{\partial^{n} F}{\partial t^{n}}\right|_{t=0} & =\sum_{n_{1}, \cdots, n_{q}} \sum_{k_{1}, \cdots, k_{q}} \frac{\left(n_{1} k_{1}+\cdots+n_{q} k_{q}\right) !}{n_{1} ! \cdots n_{q} ! k_{1}^{n_{1}} \cdots k_{q}^{n_{q}}}\left(\frac{s^{k_{1}}}{1-s^{k_{1}}}\right)^{n_{1}} \cdots\left(\frac{s^{k_{q}}}{1-s^{k_{q}}}\right)^{n_{q}} \delta_{n, n_{1} k_{1}+\cdots n_{q} k_{q}} \\
& =\sum_{n_{1}, \cdots, n_{q}} \sum_{k_{1}, \cdots, k_{q}} \frac{n ! s^{n}}{n_{1} ! \cdots n_{q} ! k_{1}^{n_{1}} \cdots k_{q}^{n_{q}}}\left(\frac{1}{1-s^{k_{1}}}\right)^{n_{1}} \cdots\left(\frac{1}{1-s^{k_{q}}}\right)^{n_{q}} \delta_{n, n_{1} k_{1}+\cdots n_{q} k_{q}}
\end{aligned}
$$

Notice that this is a sum over conjugacy classes of $S_{n}$. The conjugacy class collects permutations with $n_{q} k_{q}$-cycles. This interpretation follows because the coefficient

$$
\frac{n !}{n_{1} ! \cdots n_{q} ! k_{1}^{n_{1}} \cdots k_{q}^{n_{q}}}
$$

is the order of the conjugacy class. There is a factor of $\left(1-s^{k}\right)^{-1}$ for each $k$-cycle in the permutation. Here are a few motivational examples

$$
\begin{aligned}
\left.\frac{\partial F}{\partial t}\right|_{t=0} & =\left.\frac{s}{1-s} \quad \frac{\partial^{2} F}{\partial t^{2}}\right|_{t=0}=\frac{s^{2}}{(1-s)^{2}}+\frac{s^{2}}{1-s^{2}}=\frac{2 s^{2}}{(1-s)\left(1-s^{2}\right)} \\
\left.\frac{\partial^{3} F}{\partial t^{3}}\right|_{t=0} & =\frac{s^{3}}{(1-s)^{3}}+3 \frac{s^{2}}{(1-s)\left(1-s^{2}\right)}+\frac{2 s^{3}}{1-s^{3}}=\frac{6 s^{3}}{(1-s)\left(1-s^{2}\right)\left(1-s^{3}\right)}
\end{aligned}
$$


It is easy to identify the above expressions: recall the lowest weight discrete series irrep of $\mathrm{SL}(2)$, denoted $V_{1}$, has character

$$
\chi_{1}(s)=\operatorname{Tr}_{V_{1}}\left(s^{L_{0}}\right)=\frac{s}{1-s}
$$

It then follows that $\left(P_{[n]}\right.$ projects onto the symmetric irrep i.e. a single row of $n$ boxes)

$$
\begin{aligned}
\left.\frac{\partial F}{\partial t}\right|_{t=0} & =\frac{s}{1-s} \\
& =\chi_{1}(s) \\
\left.\frac{1}{2 !} \frac{\partial^{2} F}{\partial t^{2}}\right|_{t=0} & =\frac{s^{2}}{2(1-s)^{2}}+\frac{s^{2}}{2\left(1-s^{2}\right)}=\operatorname{Tr}\left(P_{[2]} s^{L_{0}}\right) \\
& =\operatorname{Tr}_{\operatorname{Sym}\left(V_{1}^{\otimes 2}\right)}\left(s^{L_{0}}\right) \\
\left.\frac{1}{3 !} \frac{\partial^{3} F}{\partial t^{3}}\right|_{t=0} & =\frac{s^{3}}{3 !(1-s)^{3}}+\frac{3 s^{2}}{3 !(1-s)\left(1-s^{2}\right)}+\frac{2 s^{3}}{3 !\left(1-s^{3}\right)}=\operatorname{Tr}\left(P_{[3]} s^{L_{0}}\right) \\
& =\operatorname{Tr}_{\operatorname{Sym}\left(V_{1}^{\otimes 3}\right)}\left(s^{L_{0}}\right)
\end{aligned}
$$

This interpretation follows for general $n$ as proved in (3.25). Thus the general formula is

$$
\left.\frac{1}{n !} \frac{\partial^{n} F}{\partial t^{n}}\right|_{t=0}=\operatorname{Tr}\left(P_{[n]} s^{L_{0}}\right)=\frac{s^{n}}{(1-s)\left(1-s^{2}\right)\left(1-s^{3}\right) \cdots\left(1-s^{n}\right)}
$$

where the last equality follows from eq. (47) of [15], where these SL(2) sector primaries were studied in the language of oscillators. Consequently we have

$$
G_{n}^{\max }(s)=\left.\frac{(1-s)}{n !} \frac{\partial^{n} F}{\partial t^{n}}\right|_{t=0}=\frac{s^{n}}{\left(1-s^{2}\right)\left(1-s^{3}\right) \cdots\left(1-s^{n}\right)}
$$

Note the close connection between counting leading twist primaries and the multiplicities of $V_{\Lambda=n+k}^{\mathrm{SL}(2)} \otimes V_{[n]}^{S_{n}}$, which is given by the coefficient of $q^{k}$ in

$$
\prod_{i=2}^{n} \frac{1}{1-q^{i}}
$$

The result (3.34) was also recently obtained in [26].

There are three other sectors of primaries that are closely related to this one: polynomials in $\bar{z}$ correspond to primaries of the form $[n+q,-q,-q]$, polynomials in $w$ to primaries of the form $[n+q, q,-q]$ and polynomials in $\bar{w}$ to primaries of the form $[n+q,-q, q]$.

\subsection{Extremal primaries}

We now come to a more general class of primaries with charges

$$
\Delta=n+q \quad ; \quad J_{3}^{L}=\frac{q}{2}
$$


The charge $J_{3}^{R}$, which is part of $\mathrm{SU}(2)_{R}$, is not constrained. These primary operators belong to complete multiplets of $\mathrm{SU}(2)_{R}$. They correspond polynomials constructed using the pair of complex variables $z_{I}, w_{I}$. This is clear from inspection of the charges in (2.28). Translating from the polynomial representation back to the usual scalar field representation, this corresponds to the fact that all primaries are constructed using only two components of the momentum four vector operator. The two components are complex linear combinations of the (hermitian) $P_{\mu}$. Arguing as we did in the previous section, we introduce a generating function $G_{n}^{z, w}(s, x, y)$, which is now given by

$$
G_{n}^{z, w}(s, x, y)=\left[\left(1-\frac{1}{y}\right) Z_{n}^{z, w}(s, x, y)\right]_{\geq}
$$

where $Z_{n}(s, x, y)$ is obtained from

$$
\begin{aligned}
\prod_{q=0}^{\infty} \prod_{m=0}^{q} \frac{1}{\left(1-t s^{q+1} x^{q / 2} y^{m-q / 2}\right)} & =\sum_{n=0}^{\infty} t^{n} \chi_{n}(s, x, y) \\
Z_{n}^{z, w}(s, x, y) & =(1-s \sqrt{x y})(1-s \sqrt{x / y}) \chi_{n}(s, x, y)
\end{aligned}
$$

The two brackets multiplying $Z_{n}(s, x, y)$ in $(3.37)$ is a consequence of the fact that two components of the momentum four vector are used when constructing the primaries. From (3.38) it is clear that we are selecting the state from the $J_{3, L}$ multiplet (recorded using the variable $x$ ) with the highest spin. The product over $m$ in (3.38) indicates that all the states in the $J_{3, R}$ multiplet are counted. The factor of $(1-1 / y)$ as well as the instruction (indicated with the subscript $\geq$ in (3.37)) to keep only positive powers of $y$ ensures that we count each $\mathrm{SU}(2)_{R}$ spin multiplet once. It is clear that the expansion of (3.37) has only positive powers of $x$. This is a consequence of the fact that we kept only one state from each $\mathrm{SU}(2)_{L}$ multiplet.

It is again possible to derive closed expressions for the generating functions $Z_{n}^{z, w}(s, x, y)$ and $G_{n}^{z, w}(s, x, y)$. Introduce the functions

$$
\begin{aligned}
F_{2}(t, s, x, y) & =\prod_{q=0}^{\infty} \prod_{m=0}^{q} \frac{1}{1-t s^{q+1} x^{\frac{q}{2}} y^{m-\frac{q}{2}}}=\sum_{n=0}^{\infty} t^{n} \chi_{n}(s, x, y) \\
\frac{\partial}{\partial t} F_{2}(t, s, x, y) & =\sum_{q=0}^{\infty} \sum_{m=0}^{q} \frac{s^{q+1} x^{\frac{q}{2}} y^{m-\frac{q}{2}}}{1-t s^{q+1} x^{\frac{q}{2}} y^{m-\frac{q}{2}}} F_{2}(t, s, x, y) \equiv f_{1}(t, s, x, y) F_{2}(t, s, x, y) \\
f_{k}(t, s, x, y) & \equiv \frac{\partial^{k-1} f_{1}}{\partial t^{k-1}}=(k-1) ! \sum_{q=0}^{\infty} \sum_{m=0}^{q} \frac{s^{k q+k} x^{\frac{q k}{2}} y^{k m-\frac{k q}{2}}}{\left(1-t s^{q+1} x^{\frac{q}{2}} y^{m-\frac{q}{2}}\right)^{k}}
\end{aligned}
$$

It is simple to establish that $F_{2}(0, s, x, y)=1$ and

$$
f_{k}(0, s, x, y)=s^{k}(k-1) ! \frac{1}{1-s^{k} x^{\frac{k}{2}} y^{\frac{k}{2}}} \frac{1}{1-s^{k} x^{\frac{k}{2}} y^{-\frac{k}{2}}}
$$

Exactly as above we have

$$
\left.\frac{\partial^{n} F_{2}}{\partial t^{n}}\right|_{t=0}=\sum_{n_{1}, \cdots, n_{q}} \sum_{k_{1}, \cdots, k_{q}} \frac{\left(n_{1} k_{1}+\cdots+n_{q} k_{q}\right) !}{n_{1} ! \cdots n_{q} !\left(k_{1} !\right)^{n_{1}} \cdots\left(k_{q} !\right)^{n_{q}}} f_{k_{1}}^{n_{1}} \cdots f_{k_{q}}^{n_{q}} \delta_{n, n_{1} k_{1}+\cdots n_{q} k_{q}}
$$


Inserting the formulas for the $f_{k}$ 's and streamlining the notation by using $a=s \sqrt{x y}$ and $b=s \sqrt{\frac{x}{y}}$, we find

$$
\begin{aligned}
\left.\frac{1}{n !} \frac{\partial^{n} F_{2}}{\partial t^{n}}\right|_{t=0}= & \sum_{n_{1}, \cdots, n_{q}} \sum_{k_{1}, \cdots, k_{q}} \frac{s^{n}}{n_{1} ! \cdots n_{q} ! k_{1}^{n_{1}} \cdots k_{q}^{n_{q}}}\left(\frac{1}{\left(1-a^{k_{1}}\right)\left(1-b^{k_{1}}\right)}\right)^{n_{1}} \\
& \cdots\left(\frac{1}{\left(1-a^{k_{q}}\right)\left(1-b^{k_{q}}\right)}\right)^{n_{q}} \delta_{n, n_{1} k_{1}+\cdots n_{q} k_{q}} \\
= & \chi_{n}(s, x, y)
\end{aligned}
$$

The expression for $Z_{n}(s, x, y)$ now follows from (3.39).

It is not easy to proceed for general $n$, but it is straight forwards to obtain explicit formulas once a specific $n$ is chosen. For example, the final result for $n=3$ fields is

$$
Z_{3}^{z, w}(s, x, y)=\frac{s^{3}\left(s^{6} x^{3}+s^{4} x^{2}+s^{2} x+1+s^{3} x^{\frac{3}{2}}\left(\sqrt{y}+\frac{1}{\sqrt{y}}\right)\right)}{\left(1-s^{2} x y\right)\left(1-s^{3}(x y)^{\frac{3}{2}}\right)\left(1-s^{2} \frac{x}{y}\right)\left(1-\frac{s^{3} x^{\frac{3}{2}}}{y^{\frac{3}{2}}}\right)}
$$

To extract spin multiplets, we need to compute

$$
G_{3}^{z, w}(z, w)=\left[Z_{3}(s, x, y)\left(1-\frac{1}{y}\right)\right]_{\geq}=\frac{1}{2 \pi i} \oint_{C} d z \frac{\left(1-\frac{1}{z^{2}}\right) Z_{3}\left(s, x, z^{2}\right)}{z-\sqrt{y}}
$$

The contour $C$ must have a radius larger than $\sqrt{y}$. We assume that $s, x$ and $y$ are all less than one so that the expansion of $Z_{3}^{z, w}(s, x, y)$ converges. Thus, we can take $C$ to be the unit circle. The integrand has poles at $z= \pm s \sqrt{x}, z=\sqrt{y}, z= \pm \frac{1}{s \sqrt{x}}, z=-\frac{s \sqrt{x}}{2}(1 \pm i \sqrt{3})$ and $z=-\frac{(1 \pm i \sqrt{3})}{2 s \sqrt{x}}$. To compute the integral we need to pick up the residues from the poles at $z= \pm s \sqrt{x}, z=\sqrt{y}$, and $z=-\frac{s \sqrt{x}}{2}(1 \pm i \sqrt{3})$. We obtain

$$
G_{3}^{z, w}(z, w)=\frac{s^{3}\left(1-s^{10} x^{5} y^{3}\right)}{\left(1-s^{4} x^{2}\right)\left(1-s^{3} \sqrt{x^{3} y^{3}}\right)\left(1-s^{2} x y\right)\left(1-s^{5} x^{\frac{5}{2}} y^{\frac{3}{2}}\right)}
$$

It is easy to check, using mathematica, that this expression has the correct expansion. The check tests that the expansion, as a polynomial about $s=0$, of the above generating function matches the counting following from the expansion of the function appearing in (3.13).

Consider next the final result for $n=4$ fields, which is

$$
\begin{aligned}
& Z_{4}^{z, w}(s, x, y)=\left.\frac{1}{4 !} \frac{\partial^{4} F_{2}}{\partial t^{4}}\right|_{t=0} \\
& =\frac{s^{4} Q(s, x, y)}{\left(s^{2} x-y\right)^{2}\left(1-s^{2} x y\right)^{2}\left(s^{2} x+y\right)\left(-s^{3} x^{\frac{3}{2}}+y^{\frac{3}{2}}\right)\left(1+s^{2} x y\right)\left(1-s^{3} x^{\frac{3}{2}} y^{\frac{3}{2}}\right)} \\
& Q(s, x, y)=y^{\frac{7}{2}}\left(y+s^{2} x y+s^{10} x^{5} y+s^{12} x^{6} y+s^{3} x^{\frac{3}{2}} y^{\frac{1}{2}}(1+y)+s^{5} x^{\frac{5}{2}} y^{\frac{1}{2}}(1+y)\right. \\
& +s^{7} x^{\frac{7}{2}} y^{\frac{1}{2}}(1+y)+s^{9} x^{\frac{9}{2}} y^{\frac{1}{2}}(1+y)+s^{4} x^{2}(1+y)^{2} \\
& \left.+s^{6} x^{3}(1+y)^{2}+s^{8} x^{4}(1+y)^{2}\right)
\end{aligned}
$$


To extract spin multiplets, we again need to compute

$$
G_{4}^{z, w}(s, x, y)=\left[Z_{4}^{z, w}(s, x, y)\left(1-\frac{1}{y}\right)\right]_{\geq}=\frac{1}{2 \pi i} \oint_{C} d z \frac{\left(1-\frac{1}{z^{2}}\right) Z_{4}^{z, w}\left(s, x, z^{2}\right)}{z-\sqrt{y}}
$$

The contour $C$ must again have a radius larger than $\sqrt{y}$, so we again choose the unit circle $|z|=1$. The integrand has poles at $z= \pm s \sqrt{x}, z=\sqrt{y}, z= \pm \frac{1}{s \sqrt{x}}, z= \pm i s x, z=$ $-\frac{s \sqrt{x}}{2} i(1 \pm i \sqrt{3}), z= \pm \frac{i}{s x}$ and $z=-\frac{(1 \pm i \sqrt{3})}{2 s \sqrt{x}}$. The integral above receives contributions from the poles at $z= \pm s \sqrt{x}, z=\sqrt{y}, z= \pm i s x$, and $z=-\frac{s \sqrt{x}}{2} i(1 \pm i \sqrt{3})$. We obtain

$$
G_{4}^{z, w}(s, x, y)=\frac{s^{4} R(s, x, y)}{\left(1-s^{2} x y\right)\left(1-s^{3} x^{\frac{3}{2}} y^{\frac{3}{2}}\right)\left(1-s^{4} x^{2} y^{2}\right)\left(1-s^{4} x^{2}\right)\left(1-s^{6} x^{3}\right)\left(1-s^{8} x^{4}\right)}
$$

where

$$
\begin{aligned}
R(s, x, y)=1+ & s^{5} x^{\frac{5}{2}}\left(\sqrt{y}+s^{3} x^{\frac{3}{2}} y+s^{5} x^{\frac{5}{2}} y+y^{3}-s^{6} x^{3} y^{\frac{5}{2}}-s^{8} x^{4} y^{\frac{5}{2}}-s^{16} x^{8} y^{\frac{7}{2}}\right. \\
& -s^{11} x^{\frac{11}{2}} y^{2}(1+y)+s^{7} x^{\frac{7}{2}}\left(1-y^{2}\right)+s^{4} x^{2} y^{\frac{3}{2}}\left(1-y^{2}\right)+s^{2} x \sqrt{y}\left(1+y^{2}\right) \\
& \left.-s^{9} x^{\frac{9}{2}} y\left(1+y^{2}\right)-s^{10} x^{5} y^{\frac{3}{2}}\left(1+y-y^{2}\right)-s \sqrt{x}\left(1-y-y^{2}\right)\right)
\end{aligned}
$$

It is again easy to check, using mathematica, that this expression does indeed have the correct expansion.

There are other sectors of primaries that are slight variations of the extremal sector studied in this section. Polynomials in $z_{I}, \bar{w}_{I}$ correspond to primaries with $\left(\Delta=n+q, J_{3}^{R}=\right.$ $q)$. Polynomials in $\bar{z}_{I}, w_{I}$ correspond to primaries with $\left(\Delta=n+q, J_{3}^{R}=-q\right)$. Polynomials in $\bar{z}_{I}, \bar{w}_{I}$ correspond to primaries with $\left(\Delta=n+q, J_{3}^{L}=-q\right)$.

\section{Construction and construction with symmetric groups}

In this section we would like to provide construction formulas for the extremal primaries we have counted in section 3. To accomplish this the polynomial representation of $\mathrm{SO}(4,2)$ introduced in section 2 will play a central role. These polynomials are constructed using the coordinates $x_{\mu}^{I}, I=1, \ldots, n$ which admit a natural action of $S_{n}$. Constructing primaries then amounts to constructing polynomials that are consistent with (2.25). The first of (2.25) can be satisfied by constructing $n-1$ translationaly invariant "relative coordinates" out of the $x_{\mu}^{I}$. This construction is not unique. Following [15], a particularly convenient choice makes use of the variables

$$
X_{\mu}^{(a)}=\frac{1}{\sqrt{a(a+1)}}\left(x_{\mu}^{1}+\cdots+x_{\mu}^{a}-a x_{\mu}^{a+1}\right)
$$

These variables are in the $[n-1,1]$ irrep of $S_{n}$. To satisfy the second of $(2.25)$ we need to build polynomials that are harmonic. In terms of complex coordinates the Laplacian is

$$
\sum_{\mu} \frac{\partial}{\partial x_{\mu}^{I}} \frac{\partial}{\partial x_{\mu}^{I}}=\frac{\partial}{\partial z^{I}} \frac{\partial}{\partial \bar{z}^{I}}+\frac{\partial}{\partial w^{I}} \frac{\partial}{\partial \bar{w}^{I}}
$$


It is clear that we can build harmonic polynomials by considering polynomials that are functions only of the $z^{I}$, which gives the leading twist primaries, or that are functions of the $z^{I}$ and $w^{I}$, which gives the leading left twist primaries considered in section 3.3. The harmonic constraint is the only constraint that is not first order. By replacing this with a holomorphic constraint, which is first order, the resulting problem entails finding families of polynomials that obey first order equations. This implies that the problem will now have a natural ring structure, something which will be visible in our construction. The final constraint that needs to be obeyed is that the polynomials are $S_{n}$ invariants. The counting formulas we derived in the previous section will give valuable insight into how to handle this final constraint.

\subsection{Leading twist primaries}

Specializing to $n=3$ and employing complex variables, we have

$$
Z^{(1)}=\frac{z^{1}-z^{2}}{\sqrt{2}} \quad Z^{(2)}=\frac{z^{1}+z^{2}-2 z^{3}}{\sqrt{6}}
$$

plus the obvious formulas for $\bar{Z}^{(a)}, W^{(a)}$ and $\bar{W}^{(a)}$. The nice thing about these variables is that $S_{n}$ acts on these variables with Young's orthogonal representation of $[n-1,1]$, i.e. for $n=3$ we have [27]

$$
\Gamma_{\square}((12))=\left[\begin{array}{cc}
-1 & 0 \\
0 & 1
\end{array}\right] \quad \Gamma_{\square}((23))=\left[\begin{array}{cc}
\frac{1}{2} & \frac{\sqrt{3}}{2} \\
\frac{\sqrt{3}}{2} & -\frac{1}{2}
\end{array}\right]
$$

The remaining elements of the group can be generated using these two. When acting on a product of variables, say $Z^{\left(a_{1}\right)} Z^{\left(a_{2}\right)} \cdots Z^{\left(a_{k}\right)}$ we have

$$
\Gamma_{k}(\sigma)=\Gamma_{\boxplus}(\sigma) \times \cdots \times \Gamma_{\boxplus}(\sigma)
$$

where we take a tensor product (the usual Kronecker product) of $k$ copies of the matrices of the hook irrep. Any polynomial in the hook variables automatically obeys (2.25). Thus, all that is left is to project to $S_{n}$ invariants in $V_{H}^{\otimes k}$. We can build these by acting with the projector from the tensor product of $k$ copies of the hook onto the trivial irrep

$$
P_{\text {m }}=\frac{1}{3 !} \sum_{\sigma \in S_{3}} \Gamma_{k}(\sigma)
$$

Acting on $Z^{\otimes k}$ we obtain an expression of the form $\sum_{i} \hat{n}_{i} P_{i}(z)$ where $\hat{n}_{i}$ are unit vectors inside the carrier space of $\nabla^{\otimes k}$ and $P_{i}(z)$ are the polynomials that can be translated into primary operators.

It is useful to consider a few examples. Acting with the projector (4.5) on the tensor product of $k$ copies of the hook, we find

$$
P_{a_{1} a_{2} \cdots a_{k}}=\sum_{\sigma \in S_{3}} \Gamma_{k}(\sigma)_{a_{1} a_{2} \cdots a_{k}, b_{1} b_{2} \cdots b_{k}} Z^{\left(b_{1}\right)} Z^{\left(b_{2}\right)} \cdots Z^{\left(b_{k}\right)}
$$


It is simple to implement this projector in mathematica. For $k=1$ we find $P_{a_{1}}=0$. For $k=2$ the projector is

$$
P_{a_{1} a_{2}}=\left(\left(z_{1}-z_{2}\right)^{2}+\left(z_{1}-z_{3}\right)^{2}+\left(z_{2}-z_{3}\right)^{2}\right)\left[\begin{array}{c}
\frac{1}{6} \\
0 \\
0 \\
\frac{1}{6}
\end{array}\right]
$$

so the invariant polynomial is

$$
P(z)=\left(\left(z_{1}-z_{2}\right)^{2}+\left(z_{1}-z_{3}\right)^{2}+\left(z_{2}-z_{3}\right)^{2}\right)
$$

By inspection, this obviously obeys (2.25). For $k=3$ the projector is

$$
P_{a_{1} a_{2} a_{3}}=\left(\left(z_{1}+z_{2}-2 z_{3}\right)\left(z_{1}+z_{3}-2 z_{2}\right)\left(z_{2}+z_{3}-2 z_{1}\right)\right)\left[\begin{array}{c}
0 \\
\frac{-1}{6 \sqrt{6}} \\
\frac{-1}{6 \sqrt{6}} \\
0 \\
\frac{-1}{6 \sqrt{6}} \\
0 \\
0 \\
\frac{1}{6 \sqrt{6}}
\end{array}\right]
$$

so the invariant polynomial is

$$
P(z)=\left(\left(z_{1}+z_{2}-2 z_{3}\right)\left(z_{1}+z_{3}-2 z_{2}\right)\left(z_{2}+z_{3}-2 z_{1}\right)\right)
$$

This polynomial again obeys (2.25). Finally, for $k=4$ the projector is

$$
P_{a_{1} a_{2} a_{3} a_{4}}=\left(\left(z_{1}-z_{2}\right)^{4}+\left(z_{1}-z_{3}\right)^{4}+\left(z_{2}-z_{3}\right)^{4}\right)\left[\begin{array}{c}
\frac{1}{12} \\
0 \\
0 \\
\frac{1}{36} \\
0 \\
\frac{1}{36} \\
\frac{1}{36} \\
0 \\
0 \\
\frac{1}{36} \\
\frac{1}{36} \\
0 \\
\frac{1}{36} \\
0 \\
0 \\
\frac{1}{12}
\end{array}\right]
$$


so the invariant polynomial is

$$
P(z)=\left(\left(z_{1}-z_{2}\right)^{4}+\left(z_{1}-z_{3}\right)^{4}+\left(z_{2}-z_{3}\right)^{4}\right)
$$

This clearly obeys (2.25), so this is again the correct answer.

The polynomials we construct in this way will obey the conditions spelled out in (2.25). In fact, they obey an even stronger linear condition

$$
\partial_{\bar{z}^{I}} P(\vec{z})=0=\partial_{\bar{w}^{I}} P(\vec{z})
$$

which imply the Laplacian constraint. As a result, taking all possible values of $k$ we find that the polynomials constructed in this way exhibit a highly non-trivial structure enjoyed by the leading twist primaries: the polynomials $P_{i}(z)$ are a finitely generated polynomial ring. The counting formula (3.34) gives the Hilbert series for holomorphic functions on $\left(\mathbb{C}^{n} / \mathbb{C}\right) / S_{n}$. The quotient by $\mathbb{C}$ sets the center of mass momentum of the many body wave function to zero as dictated by the first of $(2.25)$. The orbifold by $S_{n}$ implements the last of (2.25). The counting formula (3.34) implies that the ring has $n-1$ generators. These generators are given by constructing the $n-1$ possible independent $S_{n}$ invariants out of the hook variables introduced in (4.1). For example, for $n=2$ fields the polynomials are generated by $\left(z_{1}-z_{2}\right)^{2}$. The polynomials corresponding to primaries are

$$
\left(z_{1}-z_{2}\right)^{2 k}
$$

Using (2.19) it is easy to see that (these vanish if $s$ is odd)

$$
\begin{aligned}
O_{s} & =\left(z_{1}-z_{2}\right)^{s} \\
& \leftrightarrow \frac{s !}{2^{s}} \sum_{k=0}^{s} \frac{(-1)^{k}}{(k !(s-k) !)^{2}} \partial_{z}^{s-k} \phi \partial_{z}^{k} \phi
\end{aligned}
$$

reproducing the higher spin currents, given for example in [28]. For $n=3$ fields the ring of polynomials that correspond to primary operators is generated by

$$
\left(z_{1}-z_{2}\right)^{2}+\left(z_{1}-z_{3}\right)^{2}+\left(z_{2}-z_{3}\right)^{2}
$$

and

$$
\left(z_{1}+z_{2}-2 z_{3}\right)\left(z_{3}+z_{2}-2 z_{1}\right)\left(z_{1}+z_{3}-2 z_{2}\right)
$$

In general, the generators of the ring are a product of the variables $Z^{(a)}$ introduced above, such that the product is $S_{n}$ invariant. For $n=4$ the ring is generated by $\left(z_{1}-z_{2}\right)\left(z_{2}-z_{1}\right)+$ $\cdots,\left(z_{1}+z_{2}-2 z_{3}\right)\left(z_{3}+z_{2}-2 z_{1}\right)\left(z_{1}+z_{3}-2 z_{2}\right)+\cdots$ and $\left(z_{1}+z_{2}+z_{3}-3 z_{4}\right)\left(z_{3}+z_{2}+z_{4}-\right.$ $\left.3 z_{1}\right)\left(z_{1}+z_{3}+z_{4}-3 z_{2}\right)\left(z_{1}+z_{2}+z_{4}-3 z_{3}\right)$, where $\cdots$ stand for terms that must be summed to obtain an $S_{4}$ invariant. The ring structure that has appeared is rather interesting. The product on the ring is simply multiplication of polynomials. This is a natural product in the polynomial language, but is highly non-trivial in the original CFT description. A natural guess would be that this is somehow connected to the OPE of primaries, which is 
the natural product on the primaries of the CFT. However, this cannot be correct because the polynomial ring exists for a fixed number $n$. Thus, in terms of the CFT language, the ring multiplication is a product between two primaries, each of which has $n$ fields, and the result is again a primary with $n$ fields. The operator product of two local operators, each containing $n$ fields, is a sum of operators containing $2 n-2 k$ fields with $k=0,1, \ldots, n$. For odd $n$ the product of elements of the ring gives an operator with $n$ fields. This product can therefore not even be a subalgebra of the CFT operator product algebra. This product and the associated ring structure of primary fields in free CFT4 appears to be a genuinely new structure, not previously noticed.

A natural question to ask is whether or not these primary operators are orthogonal. We can translate any polynomial into an operator and then compute the two point function of the operator. The computation can also be carried out by a judicious choice of an inner product for the polynomial. For example, consider the correlator

$$
\left\langle\partial_{z}^{k} \phi(x) \partial_{z^{\prime}}^{l} \phi\left(x^{\prime}\right)\right\rangle=(-1)^{k}(k+l) ! \frac{\left(\bar{z}-\bar{z}^{\prime}\right)^{k+l}}{\left(\left|z-z^{\prime}\right|^{2}+\left|w-w^{\prime}\right|^{2}\right)^{k+l+1}}
$$

Everything in the above result is determined by conformal invariance, except the overall number $=(-1)^{k}(k+l)$ !. Recalling that $z^{n}$ translates into $\frac{1}{n !} \partial_{z}^{n}$, this number can be computed if we use the following inner product for the polynomials

$$
\left\langle z^{k} z^{\prime l}\right\rangle_{p}=(-1)^{k} \frac{(k+l) !}{k ! l !}
$$

Notice that the norm following from this inner product is not positive definite. For $n$ fields we have polynomials in $z_{k}$ for the primary at $x$ and in $z_{k}^{\prime}$ for the primary at $x^{\prime}$, with $k=1, \ldots, n$. In this more general setting, the inner product is

$$
\left\langle\prod_{k=1}^{n} z_{k} p_{k} \prod_{l=1}^{n} z_{l}^{\prime q_{l}}\right\rangle_{p}=\prod_{k=1}^{n}(-1)^{p_{k}} \frac{\left(p_{k}+q_{k}\right) !}{p_{k} ! q_{k} !}
$$

In addition, due to Wick's theorem, there are a total of $n$ ! Wick contractions contributing, which introduces a factor of $n$ !. In the end, if polynomials $P_{i}$ of degree $k_{i}$ in $n$ variables translate into primaries $\mathcal{O}_{i}$ constructed from $n$ fields with dimension $n+k_{i}$, then we have

$$
\left\langle\mathcal{O}_{i}(x) \mathcal{O}_{j}\left(x^{\prime}\right)\right\rangle=\frac{c_{i j}\left(\bar{z}-\bar{z}^{\prime}\right)^{k_{i}+k_{j}}}{\left(\left|z-z^{\prime}\right|^{2}+\left|w-w^{\prime}\right|^{2}\right)^{k_{i}+k_{j}+n}}
$$

with

$$
c_{i j}=n !\left\langle P_{i}\left(z_{k}\right) P_{j}\left(z_{k}^{\prime}\right)\right\rangle_{p}
$$

Using the above formulas, it is easy to check that primary operators with different dimensions are orthogonal, as they must be. Further, we also see that although our ring of primaries is a basis, the operators in the basis are not orthogonal. 


\subsection{Extremal primaries}

The above construction is easily extended to the other classes of extremal primaries we have counted. The leading left or right twist class is provided by polynomials in two holomorphic coordinates, $z$ and $w$. Consider polynomials of degree $k$ in $Z$ and of degree $l$ in $W$, with $Z, W$ the hook variables transforming in the hook representation $V_{H}$ of $S_{n}$, described by a Young diagram with row lengths $[n-1,1]$. These polynomials belong to a subspace of $V_{H}^{\otimes k} \otimes V_{H}^{\otimes l}$ of $S_{n}$. To characterize this subspace using representation theory, start with the decompositions in terms of $S_{n} \times S_{k}$ irreps

$$
\begin{aligned}
& V_{H}^{\otimes k}=\bigoplus_{\Lambda_{1} \vdash n, \Lambda_{2} \vdash k} V_{\Lambda_{1}}^{\left(S_{n}\right)} \otimes V_{\Lambda_{2}}^{\left(S_{k}\right)} \otimes V_{\Lambda_{1}, \Lambda_{2}}^{C o m\left(S_{n} \times S_{k}\right)} \\
& V_{H}^{\otimes l}=\bigoplus_{\Lambda_{3} \vdash n, \Lambda_{4} \vdash l} V_{\Lambda_{3}}^{\left(S_{n}\right)} \otimes V_{\Lambda_{4}}^{\left(S_{l}\right)} \otimes V_{\Lambda_{3}, \Lambda_{4}}^{C o m\left(S_{n} \times S_{l}\right)}
\end{aligned}
$$

$\operatorname{Com}\left(S_{n} \times S_{k}\right)$ is the algebra of linear operators on $V_{H}^{\otimes k}$ which commute with $S_{n} \times S_{k}$. The tensor product $V_{H}^{\otimes k} \otimes V_{H}^{\otimes l}$ is a representation of

$$
\mathbb{C}\left(S_{n}\right) \otimes \mathbb{C}\left(S_{k}\right) \otimes \mathbb{C}\left(S_{n}\right) \otimes \mathbb{C}\left(S_{l}\right)
$$

These decompositions (4.22) have been studied in detail in [15] where they were used to construct BPS states of $\mathcal{N}=4$ SYM. In the application we consider here, the $Z$ and $W$ variables are commuting which implies that they are in the trivial rep $\Lambda_{2} \otimes \Lambda_{4}=[k] \otimes[l]$ of $S_{k} \times S_{l}$. The multiplicity with which a given $S_{n} \times S_{k}$ irrep $\left(\Lambda_{1}, \Lambda_{2}\right)$ appears is given by the dimension of the irrep of the commutants $\operatorname{Com}\left(S_{n} \times S_{l}\right)$ in $V_{H}^{\otimes k}$. We want to project to states in $V_{H}^{\otimes k} \otimes V_{H}^{\otimes l}$ which are invariant under the diagonal $\mathbb{C}\left(S_{n}\right)$ in the algebra (4.23). This constrains $\Lambda_{3}=\Lambda_{1}$. Thus we find that the number of $S_{k} \times S_{l} \times S_{n}$ invariants is

$$
\sum_{\Lambda_{1} \vdash n} \operatorname{Mult}\left(\Lambda_{1},[k] ; S_{n} \times S_{k}\right) \operatorname{Mult}\left(\Lambda_{1},[l] ; S_{n} \times S_{l}\right)
$$

The generating functions for these multiplicities have been derived in [15]. $\operatorname{Mult}\left(\Lambda_{1},[k] ; S_{n} \times S_{k}\right)$ is the coefficient of $q^{k}$ in

$$
\begin{aligned}
Z_{\mathrm{SH}}\left(q ; \Lambda_{1}\right) & =(1-q) q^{\frac{\sum_{i} c_{i}\left(c_{i}-1\right)}{2}} \prod_{b} \frac{1}{\left(1-q^{h_{b}}\right)} \\
& =\sum_{k} q^{k} Z_{\mathrm{SH}}^{k}\left(\Lambda_{1}\right)
\end{aligned}
$$

Here $c_{i}$ is the length of the $i$ 'th column in $\Lambda_{1}, b$ runs over boxes in the Young diagram $\Lambda_{1}$ and $h_{b}$ is the hook length of the box $b$. Thus, for the number of primaries constructed from $z_{i}, w_{i}$ we get

$$
\sum_{\Lambda_{1} \vdash n} Z_{\mathrm{SH}}^{k}\left(\Lambda_{1}\right) Z_{\mathrm{SH}}^{l}\left(\Lambda_{1}\right)
$$

The above integer gives the number of primaries in the free scalar theory, of weight $n+k+l$, with spin $\left(J_{3}^{L}, J_{3}^{R}\right)=\left(\frac{k+l}{2}, \frac{k-l}{2}\right)$. For the generating function $Z_{n}^{z, w}(s, x, y)$ which encodes 
all $k, l$, we have

$$
Z_{n}^{z, w}(s, x, y)=s^{n} \sum_{\Lambda_{1} \vdash n} Z_{\mathrm{SH}}\left(s \sqrt{x y}, \Lambda_{1}\right) Z_{\mathrm{SH}}\left(s \sqrt{\frac{x}{y}}, \Lambda_{1}\right)
$$

where $\Lambda_{1}$ is a partition of $n$ and we can use the formula 4.25.

We can in fact see that the above discussion is consistent with the Taylor expansion formula (3.43). We can recognise this formula as $\operatorname{Tr}\left(P_{[n]} a^{L_{0}} b^{L_{0}}\right)$ where the trace is being taken in

$$
\bigoplus_{k, l=0}^{\infty} \operatorname{Sym}^{k+l}\left(V_{H}\right)
$$

which can be identified with a tensor product of discrete irreps of $\operatorname{SL}(2)$, which we may denote as $V_{\mathrm{SL}(2)}^{\otimes n} \otimes V_{\mathrm{SL}(2)}^{\otimes n}$ : one factor corresponds to the $z$ variables and another to the $w$ variables. $P_{[n]}$ is the projector for the symmetric irrep of $S_{n}$. Factor out the trace into the separate $\mathrm{SL}(2)$ factors to get (see (3.39))

$$
\begin{aligned}
\left.\frac{1}{n !} \frac{\partial^{n} F_{2}}{\partial t^{n}}\right|_{t=0} & =\operatorname{Tr}\left(P_{[n]} a^{L_{0}} b^{L_{0}}\right) \\
& =\sum_{\Lambda_{1} \vdash n} \operatorname{Tr}\left(P_{\Lambda_{1}} a^{L_{0}}\right) \operatorname{Tr}\left(P_{\Lambda_{1}} b^{L_{0}}\right)
\end{aligned}
$$

Note also that

$$
\frac{1}{(1-a)} Z_{\mathrm{SH}}(a, \Lambda)=\operatorname{Tr}\left(P_{\Lambda} a^{L_{0}}\right)
$$

which follows by recognising that the raising operators of the SL(2) representation on $z_{1} \cdots z_{n}$ can be separated into a weight one centre of mass coordinate and the differences which span the hook representation of $S_{n}$. This demonstrates the equivalence between the Taylor expansion formula (3.43) and the $S_{n} \times S_{k} \times S_{l}$ formula (4.27). It is important to note that this is a non-trivial equivalence: both formulae are self-contained ways of calculating the multiplicities.

We have thus re-expressed our earlier Taylor expansion in a way that makes the representation theory content of the counting manifest. This structure in the counting problem can be used to provide an explicit construction formula. First we need to decompose the $Z$ and $W$ polynomials into definite $S_{n}$ irreps. The projector onto irrep $r$ from the tensor product of $k$ copies of the hook is

$$
P^{r, k}=\frac{1}{n !} \sum_{\sigma \in S_{n}} \chi_{r}(\sigma) \Gamma_{k}(\sigma)
$$

We also need the projection onto the symmetric irrep

$$
P^{k+l}=\frac{1}{n !} \sum_{\sigma \in S_{n}} \Gamma_{k+l}(\sigma)
$$


Using these two projectors, the polynomials corresponding to primaries constructed using two holomorphic variables are now given by

$$
\sum_{A} P_{A}(z, w) \hat{n}^{A}=P^{k+l} \sum_{r \vdash n}\left(P^{r, l} \times P^{r, k}\right) Z^{\otimes k} W^{\otimes l}
$$

where $\hat{n}^{A}$ are unit vectors inside the carrier space of $\square^{\otimes k+l}$ and $P_{A}(z, w)$ are the polynomials we want. In fact, the construction formula given in (4.33) constructs a larger class of polynomials than those counted in (3.37). This is because the polynomials counted in (3.37) are extremal and hence they are annihilated by $J_{+}^{R}$. We will return to this point in the discussion below.

The construction formula that has been sketched above can easily be implemented numerically. To implement (4.33), we need the projector onto irrep $r$ in the space obtained by taking the tensor product of $k$ copies of the hook

$$
P_{a_{1} \cdots a_{k}, b_{1} \cdots b_{k}}^{r}=\frac{1}{n !} \sum_{\sigma \in S_{n}} \chi_{r}(\sigma) \Gamma_{k}(\sigma)_{a_{1} a_{2} \cdots a_{k}, b_{1} b_{2} \cdots b_{k}}
$$

and we need the projection onto the symmetric irrep

$$
P_{a_{1} \cdots a_{n}, b_{1} \cdots b_{n}}=\frac{1}{n !} \sum_{\sigma \in S_{n}} \Gamma_{k}(\sigma)_{a_{1} \cdots a_{n}, b_{1} \cdots b_{n}}
$$

We find that (4.33) is now given by

$$
\begin{aligned}
\sum_{A} P_{A}(z, w) \hat{n}_{e_{1} \cdots e_{k+l}}^{A}= & P_{e_{1} \cdots e_{k+l}, a_{1} \cdots a_{k} c_{1} \cdots c_{l}} P_{a_{1} \cdots a_{k}, b_{1} \cdots b_{k}}^{r} P_{c_{1} \cdots c_{l}, d_{1} \cdots d_{l}}^{r} \\
& \times Z^{\left(b_{1}\right)} \cdots Z^{\left(b_{k}\right)} W^{\left(d_{1}\right)} \cdots W^{\left(d_{l}\right)} \\
= & \tilde{P}_{e_{1} \cdots e_{k+l}}
\end{aligned}
$$

where $\hat{n}^{A}$ are unit vectors and $P_{A}(, w z)$ are the polynomials we want. To start, consider $k=l=1$. We find

$$
\tilde{P}_{e_{1} e_{2}}=\left(-w_{3}\left(z_{1}+z_{2}-2 z_{3}\right)+w_{1}\left(2 z_{1}-z_{2}-z_{3}\right)-w_{2}\left(z_{1}-2 z_{2}+z_{3}\right)\right)\left[\begin{array}{l}
1 \\
0 \\
0 \\
1
\end{array}\right]
$$

so that the invariant polynomial is

$$
P(z, w)=-w_{3}\left(z_{1}+z_{2}-2 z_{3}\right)+w_{1}\left(2 z_{1}-z_{2}-z_{3}\right)-w_{2}\left(z_{1}-2 z_{2}+z_{3}\right)
$$

This polynomial is not extremal, which is easily verified by computing

$$
-J_{+}^{R} P(z, w)=z_{i} \frac{\partial}{\partial w_{i}} P(z, w)=\left(z_{1}-z_{2}\right)^{2}+\left(z_{1}-z_{3}\right)^{2}+\left(z_{2}-z_{3}\right)^{2}
$$

so that this is another state in the multiplet of the $k=2$ primary we built in the last section.

To focus on the extremal polynomials counted in (3.37) we must implement the constraint that these polynomials are annihilated by $J_{+}^{R}$. Towards this end, note that the 
polynomials in $Z, W$ carry a representation of $\mathrm{SU}(2)_{R}$, so that we can further decompose the polynomials according to their $\mathrm{SU}(2)_{R}$ quantum numbers. $Z, W$ form an $\mathrm{SU}(2)$ doublet with $Z$ the $+\frac{1}{2}$ state and $W$ the $-\frac{1}{2}$ state. There is an action of $S_{k+l}$ on these polynomials that commutes with $\mathrm{SU}(2)_{R}$. This $S_{k+l}$ action acts to permute the $W^{(a)}$ and $Z^{(b)}$ factors. Denote the matrix representing $\sigma \in S_{k+l}$ by $\Gamma(\sigma)$. This rep is generated by the adjacent permutations which are easy to build. Towards this end, note that swapping two factors in the tensor product is accomplished by the permutation $P$ which obeys $P x \otimes y=y \otimes x$, i.e. we have

$$
P\left[\begin{array}{l}
x_{1} y_{1} \\
x_{1} y_{2} \\
x_{2} y_{1} \\
x_{2} y_{2}
\end{array}\right]=\left[\begin{array}{l}
x_{1} y_{1} \\
x_{2} y_{1} \\
x_{1} y_{2} \\
x_{2} y_{2}
\end{array}\right] \Rightarrow P=\left[\begin{array}{llll}
1 & 0 & 0 & 0 \\
0 & 0 & 1 & 0 \\
0 & 1 & 0 & 0 \\
0 & 0 & 0 & 1
\end{array}\right]
$$

Using the adjacent permutations we can construct any $\Gamma(\sigma)$ and then any projector

$$
K^{R}=\frac{1}{(k+l) !} \sum_{\sigma \in S_{k+l}} \chi_{R}(\sigma) \Gamma(\sigma)
$$

with $\chi_{R}(\sigma)$ a symmetric group character. The label $R$ is a Young diagram with at most 2 rows. The spin of the $\mathrm{SU}(2)$ irrep that $K^{R}$ projects to is given by $\left(R_{1}-R_{2}\right) / 2$ where $R_{1}$ and $R_{2}$ are the lengths of the rows of $R$. As an example, consider $k=2=l$. The rep of $S_{4}$ we need is generated by ( $\mathbf{1}$ is the $2 \times 2$ identity)

$$
\Gamma((12))=P \otimes \mathbf{1} \otimes \mathbf{1} \quad \Gamma((23))=\mathbf{1} \otimes P \otimes \mathbf{1} \quad \Gamma((34))=\mathbf{1} \otimes \mathbf{1} \otimes P
$$

To construct the primary corresponding to $s^{7} x^{2}$ we need to project on the $\mathrm{SU}(2)_{R}$ irrep with spin zero. This is accomplished by using the projector

$$
K_{a_{1} a_{2} a_{3} a_{4}, b_{1} b_{2} b_{3} b_{4}}^{\boxplus}=\frac{1}{4 !} \sum_{\sigma \in S_{4}} \chi_{\boxplus}(\sigma) \Gamma_{a_{1} a_{2} a_{3} a_{4}, b_{1} b_{2} b_{3} b_{4}}(\sigma)
$$

It is simple to compute

$$
K_{a_{1} a_{2} a_{3} a_{4}, b_{1} b_{2} b_{3} b_{4}}^{\boxplus} \tilde{P}_{b_{1} b_{2} b_{3} b_{4}}=\left(w_{1}\left(z_{2}-z_{3}\right)+w_{2}\left(z_{3}-z_{1}\right)+w_{3}\left(z_{1}-z_{2}\right)\right)^{2}\left[\begin{array}{c}
0 \\
0 \\
0 \\
\frac{4}{3} \\
0 \\
-\frac{2}{3} \\
-\frac{2}{3} \\
0 \\
0 \\
-\frac{2}{3} \\
-\frac{2}{3} \\
0 \\
\frac{4}{3} \\
0 \\
0 \\
0
\end{array}\right]
$$


Thus the invariant polynomial is

$$
P(z, w)=\left(w_{1}\left(z_{2}-z_{3}\right)+w_{2}\left(z_{3}-z_{1}\right)+w_{3}\left(z_{1}-z_{2}\right)\right)^{2}
$$

By inspection it is obvious that this polynomial obeys the conditions (2.25) and further that it is a highest weight of $\mathrm{SU}(2)_{R}$, i.e. $J_{+}^{R} P(z, w)=0$. The above polynomial suggests a natural generalization: consider the family of polynomials indexed by the integer $n$

$$
\Psi_{n}=\left(w^{(3)}\left(\bar{z}^{(2)}-\bar{z}^{(1)}\right)+w^{(2)}\left(\bar{z}^{(1)}-\bar{z}^{(3)}\right)+w^{(1)}\left(\bar{z}^{(3)}-\bar{z}^{(2)}\right)\right)^{2 n}
$$

It is obvious that they also obey (2.25) and hence that these polynomials do correspond to primary operators. It is also clear that they are extremal, i.e. $J_{+} \Psi_{n}=0$. These primaries have spin $[2 n, 0]$ and dimension $\Delta=3+4 n$. The translation into the free field language is

$$
\begin{aligned}
\mathcal{O}_{[2 n, 0]}^{\Delta=4 n+3}=\sum_{r=0}^{2 n} \sum_{s=0}^{2 n-r} & \sum_{t=0}^{r} \sum_{u=0}^{s} \sum_{v=0}^{2 n-r-s} \frac{(2 n) !(-1)^{t+u+v}}{(r-t) ! t !(s-u) ! u !(2 n-r-s-v) ! v !} \times \\
& \times\left(P_{w}^{2 n-r-s} P_{\bar{z}}^{t+s-u} \phi\right)\left(P_{w}^{s} P_{\bar{z}}^{r-t+v} \phi\right)\left(P_{w}^{r} P_{\bar{z}}^{2 n+u-r-s-v} \phi\right)
\end{aligned}
$$

The polynomials we have constructed in (4.33) obey all of the conditions spelled out in (2.25). In fact, they again obey an even stronger linear condition

$$
\partial_{\bar{z}^{I}} P(\vec{z}, \vec{w})=0=\partial_{\bar{w}^{I}} P(\vec{z}, \vec{w})
$$

which imply the Laplacian constraint. As a result, taking all possible values of $k, l$ we find that the polynomials $P_{A}(z, w)$ are again a finitely generated polynomial ring. This is a consequence of the Leibnitz rule for the derivatives of a product of functions. The ring of polynomials that correspond to extremal primaries is the polynomial ring of holomorphic functions for

$$
\left(\mathbb{C}^{2}\right)^{n} /\left(\mathbb{C}^{2} \times S_{n}\right)
$$

In (3.46), we have computed the Hilbert series for the polynomials in two holomorphic variables, that correspond to extremal primary operators built using two scalar fields. Using generalities about Hilbert series for algebraic varieties (see [17, 18] for applications in the context of moduli spaces of SUSY gauge theories), we know that if the ring is generated by $h$ homogeneous elements of positive degrees $d_{1}, \cdots, d_{h}$, then the Hilbert series is a rational fraction

$$
H S(t)=\frac{Q(t)}{\prod_{i=1}^{h}\left(1-t^{d_{i}}\right)}
$$

where $Q$ is a polynomial with integer coefficients. Thus, we see from (3.46) that for $n=3$ the polynomials $P_{A}(z, w)$ are a finitely generated polynomial ring with 4 generators and one relation and that this space of polynomials is a complete intersection and it is 3 dimensional. 
Using this Hilbert series and the explicit constructions described above, we can identify the generators $\left(z_{i j} \equiv z_{i}-z_{j}\right)$

$$
\begin{aligned}
& G_{1}=\left(z_{12}\right)^{2}+\left(z_{13}\right)^{2}+\left(z_{23}\right)^{2} \quad \leftrightarrow s^{2} x y \\
& G_{2}=\left(z_{13}+z_{23}\right)\left(z_{31}+z_{21}\right)\left(z_{12}+z_{32}\right) \leftrightarrow s^{3} \sqrt{x^{3} y^{3}}
\end{aligned}
$$

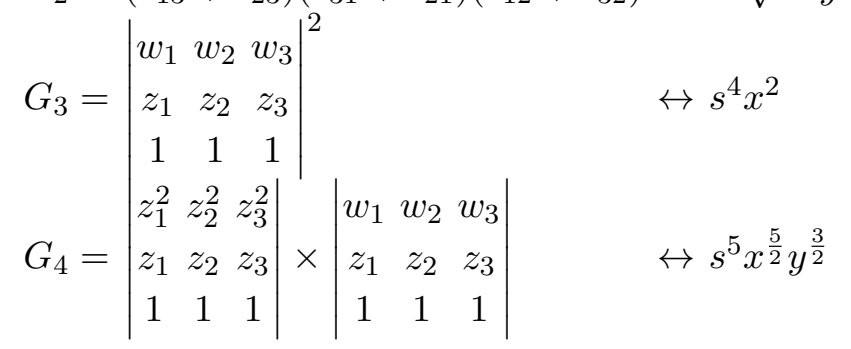

of this ring. Consider the last generator above: either of the determinants being multiplied is antisymmetric under permuting 1,2 or 1,3 or 2,3 so that the product is symmetric. The relation obeyed by these generators is easily identified

$$
27\left(G_{4}\right)^{2}+G_{3}\left(\left(G_{2}\right)^{2}-\frac{1}{2}\left(G_{1}\right)^{3}\right)=0
$$

Once again the ring structure exhibited by the polynomials implies a genuinely new structure for the extremal primary operators that was not previously recognized. The Hilbert series in more complicated situations encodes detailed information about the generators of the ring, relations between these generators, relations between the relations and so on. An example of this structure is given in appendix C.

The Hilbert series we have computed so far exhibit a palindromic property of the numerators. This can be verified for $Z_{3}^{\mathrm{z}, \mathrm{w}}(s, x, y)$ and $Z_{4}^{\mathrm{z}, \mathrm{w}}(s, x, y)$. A general property of the numerators

$$
Q_{n}(s, x, y)=\sum_{k=0}^{D} a_{k}(x, y) s^{k}
$$

is that $a_{D-k}(x, y)=a_{k}(x, y)$. A theorem due to Stanley [19] suggests that this palindromic property of the numerators implies the Calabi-Yau property of the underlying orbifolds. It is fascinating that non-trivial properties of the combinatorics of primary fields in four dimensional scalar field theory is related to the geometry of Calabi-Yau orbifolds (4.48). Motivated by this connection, we will prove this palindromic property of the numerators in the next section.

To obtain $G_{n}^{z, w}(s, x, y)$ from $Z_{n}^{z, w}(s, x, y)$, we have kept only the highest weight operator (under $\mathrm{SU}(2)$ ) from a complete spin multiplet of primary operators. Geometrically, this can be viewed as modding out by the action of $G_{+}$, generated by the $\mathrm{SU}(2)$ raising operator $J^{+}$, i.e. $G_{+}$is the unipotent group of upper triangular $2 \times 2$ matrices with 1 on the diagonal. Consequently, the Hilbert series $G_{n}(s, x, y)$ is the polynomial ring of functions for

$$
\frac{\left(\mathbb{C}^{2}\right)^{n}}{\left(\mathbb{C}^{2} \times G_{+} \times S_{n}\right)}
$$




\subsection{Palindromy properties}

The palindromic property of the Hilbert series can be stated as follows

$$
Z_{n}^{z, w}\left(q_{1}^{-1}, q_{2}^{-1}\right)=\left(q_{1} q_{2}\right)^{n-1} Z_{n}^{z, w}\left(q_{1}, q_{2}\right)
$$

In this section we will prove that our Hilbert series $Z_{n}^{z, w}\left(q_{1}, q_{2}\right)$ do indeed enjoy this transformation property.

Our starting point is the formula

$$
Z_{n}^{z, w}\left(q_{1}, q_{2}\right)=s^{n} \sum_{\Lambda \vdash n} Z_{\mathrm{SH}}\left(q_{1}, \Lambda\right) Z_{\mathrm{SH}}\left(q_{2}, \Lambda\right)
$$

where $q_{1}=s \sqrt{x y}, q_{2}=s \sqrt{x / y}$. This has the property $Z_{n}^{z, w}\left(q_{1}, q_{2}\right)=Z_{n}^{z, w}\left(q_{2}, q_{1}\right)$. The exchange of $q_{1}, q_{2}$ amounts to the inversion of $y$. Now, observe that

$$
Z_{\mathrm{SH}}\left(q^{-1}, \Lambda\right)=(-q)^{n-1} Z_{\mathrm{SH}}\left(q, \Lambda^{T}\right)
$$

This is easily demonstrated using the explicit formula (4.25) and the identity

$$
\begin{aligned}
\sum_{b} h_{b} & =\frac{1}{2}\left(\sum_{i} c_{i}\left(c_{i}+1\right)+\sum_{i} r_{i}\left(r_{i}+1\right)\right)-n \\
& =\frac{1}{2}\left(\sum_{i} c_{i}^{2}-\sum_{i} r_{i}^{2}\right)
\end{aligned}
$$

Here $c_{i}$ is the length of the $i$ 'th column and $r_{i}$ is the length of the $i$ th row. Also note that the row lengths of $\Lambda^{T}$ are the column lengths of $\Lambda$ and vice versa. The identity can be understood as follows. As we sum over hook lengths, for each column of length $c_{i}$ we have a contribution to the sum of $1+2+\cdots+c_{i}$ as we start from the bottom and go up to the top. For each row, we can similarly sum $1+2 \cdots+r_{i}$, but this over counts 1 for each box. Hence the identity. Using this result

$$
\begin{aligned}
Z_{n}^{z, w}\left(q_{1}^{-1}, q_{2}^{-1}\right) & =s^{n}\left(q_{1} q_{2}\right)^{n-1} \sum_{\Lambda \vdash n} Z_{\mathrm{SH}}\left(q_{1}, \Lambda^{T}\right) Z_{\mathrm{SH}}\left(q_{2}, \Lambda^{T}\right) \\
& =s^{n}\left(q_{1} q_{2}\right)^{n-1} \sum_{\Lambda \vdash n} Z_{\mathrm{SH}}\left(q_{1}, \Lambda\right) Z_{\mathrm{SH}}\left(q_{2}, \Lambda\right) \\
& =\left(q_{1} q_{2}\right)^{n-1} Z_{n}^{z, w}\left(q_{1}, q_{2}\right)
\end{aligned}
$$

In the last step, we used the fact that transposition is a symmetry of the set of Young diagrams. Summing over $\Lambda^{T}$ is the same as summing over $\Lambda$.

The Hilbert series $G_{n}^{z, w}(s, x, y)$ also exhibit the palindromy property. We know

$$
Z_{n}^{z, w}\left(s^{-1}, x^{-1}, y^{-1}\right)=s^{2 n-2} x^{n-1} Z_{n}^{z, w}(s, x, y)
$$

Also (CCW for counterclockwise and CW for clockwise)

$$
G_{n}^{z, w}(s, x, y)=\frac{1}{2 \pi i} \oint_{C C W} d z\left(1-\frac{1}{z^{2}}\right) Z_{n}^{z, w}\left(s, x, z^{2}\right) \frac{1}{z-\sqrt{y}}
$$


We will study $\sqrt{y} G_{n}^{z, w}(s, x, y)$ which can be written in two equivalent ways

$$
\begin{aligned}
\sqrt{y} G_{n}^{z, w}(s, x, y) & =\frac{1}{2 \pi i} \oint_{C C W} d z\left(1-\frac{1}{z^{2}}\right) Z_{n}^{z, w}\left(s, x, z^{2}\right) \frac{\sqrt{y}}{z-\sqrt{y}} \\
& =\frac{1}{2 \pi i} \oint_{C C W} d z\left(1-\frac{1}{z^{2}}\right) Z_{n}^{z, w}\left(s, x, z^{2}\right) \frac{z}{z-\sqrt{y}}
\end{aligned}
$$

Both of the representations will be needed below. Now, study

$$
\begin{aligned}
\frac{1}{\sqrt{y}} G_{n}^{z, w}\left(s^{-1}, x^{-1}, y^{-1}\right) & =\frac{1}{2 \pi i} \oint_{C C W} d z\left(1-\frac{1}{z^{2}}\right) Z_{n}^{z, w}\left(s^{-1}, x^{-1}, z^{2}\right) \frac{\frac{1}{\sqrt{y}}}{z-\frac{1}{\sqrt{y}}} \\
& =\frac{1}{2 \pi i} \oint_{C C W} d z\left(1-\frac{1}{z^{2}}\right) Z_{n}^{z, w}\left(s^{-1}, x^{-1}, z^{2}\right) \frac{1}{\sqrt{y} z-1}
\end{aligned}
$$

Now change integration variables from $z$ to $w=\frac{1}{z}$ to find

$$
\begin{aligned}
\frac{1}{\sqrt{y}} G_{n}^{z, w}\left(s^{-1}, x^{-1}, y^{-1}\right) & =-\frac{1}{2 \pi i} \oint_{C W} \frac{d w}{w^{2}}\left(1-w^{2}\right) Z_{n}^{z, w}\left(s^{-1}, x^{-1}, w^{-2}\right) \frac{w}{\sqrt{y}-w} \\
& =\frac{s^{2 n-2} x^{n-1}}{2 \pi i} \oint_{C C W} d w\left(1-\frac{1}{w^{2}}\right) Z_{n}^{z, w}(s, x, w) \frac{w}{w-\sqrt{y}} \\
& =s^{2 n-2} x^{n-1} \sqrt{y} G_{n}^{z, w}(s, x, y)
\end{aligned}
$$

\subsection{Gorenstein, Calabi-Yau and top-forms}

In this section we would like to return to the issue of the Calabi-Yau property for the permutation orbifolds relevant for the combinatorics of the primaries. Stanley's theorem [19] tells us that a Cohen Macauly ring that is an integral domain and has a palindromic Hilbert series, is a Gorenstein ring. Further, since our rings are defined over an affine space the canonical bundle in this case is trivial, establishing the Calabi-Yau property. According to [29], the rings that we consider are Cohen Macaulay because they are the quotient of a Noetherian ring $\left(\mathbb{C}^{2}\right)^{n} / \mathbb{C}^{2}$ by a reductive group $S_{n}$. However, in general, the relevant rings are not an integral domain. It is therefore not clear that we can apply Stanley's theorem to conclude that our permutation orbifolds are Calabi-Yau.

An alternative approach to demonstrating the Calabi-Yau property, is to construct a nowhere vanishing top form. To motivate the general formula, it is useful to start with some simple cases. For $n=2$ the top form

$$
\Omega^{(n-1)}(d z)=d z_{12}=d z_{1}-d z_{2}
$$

is clearly a translation invariant form on $\mathbb{C}^{2}$ so it is clearly a top form on the quotient $\mathbb{C}^{2} / \mathbb{C}$. It is odd under $S_{2}$. For $n=3$, a translation invariant, $S_{n}$-odd top form is given by

$$
\Omega^{(n-1)}(d z)=d z_{12} \wedge d z_{23}=d z_{1} \wedge d z_{2}-d z_{1} \wedge d z_{3}+d z_{2} \wedge d z_{3}
$$

For general $n$, we have

$$
\begin{aligned}
\Omega^{(n-1)}(d z) & =d z_{12} \wedge d z_{23} \wedge \cdots \wedge d z_{n-1, n} \\
& =\sum_{k=1}^{n} I_{\partial_{k}} d z_{1} \wedge d z_{2} \wedge \cdots \wedge d z_{n}
\end{aligned}
$$


The operator $I_{\partial_{k}}$ removes the $d z_{k}$ in the $n$-form and leaves an $(n-1)$-form, with a sign $(-1)^{k-1}$. In terms of these, the top forms for the orbifolds relevant for the extremal primary problem are

$$
\Omega^{(n-1)}(d z) \wedge \Omega^{(n-1)}(d w)
$$

\section{Vector model primaries: symmetry breaking $S_{2 n} \rightarrow S_{n}\left[S_{2}\right]$}

Up to now we have considered a single real scalar field. However, the methods we have developed readily apply in more general settings. For applications to holography [30], it is natural to consider the free gauged $\mathrm{O}(N)$ vector model, conjectured to be dual to higher spin gravity [31]. The scalar field is now an $\mathrm{O}(N)$ vector and primaries must be $\mathrm{O}(N)$ gauge invariants. In this section we will explain how the techniques we have developed in this article apply to the counting and construction of primaries in the gauged $\mathrm{O}(N)$ vector model.

To obtain a gauge invariant, all vector indices must be contracted. Thus, to construct a primary, we now distribute the derivatives among

$$
\phi_{I_{1}} \phi_{I_{1}} \phi_{I_{2}} \phi_{I_{2}} \cdots \phi_{I_{n}} \phi_{I_{n}}
$$

where the vector indices $I_{a}$ are summed from 1 to $N$. We no longer have an $S_{2 n}$ symmetry acting to swap the bosonic fields. The symmetry is broken to a smaller group which can swap the fields in a given contracted pair, or it can swap the pairs. This symmetry group is the wreath product $S_{n}\left[S_{2}\right]$. Thus, we don't want to project $V_{+}^{\otimes 2 n}$ onto the trivial of $S_{2 n}$ (i.e. $\operatorname{Sym}\left(V_{+}^{\otimes 2 n}\right)$ ), we rather want to project onto the trivial of $S_{n}\left[S_{2}\right]$. We will restrict attention to the case where $2 n<N$. This avoids subtleties due to finite $N$ relations, associated with the stringy exclusion principle in the context of matrix invariants. These can be dealt with using a Young diagram basis, which is left for a future discussion.

We know the character for the fundamental representation $V_{+}$of $\mathrm{SO}(4,2)$. To repeat the analysis we carried out for the free scalar, we need the character for the tensor product of $2 n$ fields, after projecting to the trivial of $S_{n}\left[S_{2}\right]$. This gives

$$
\chi_{\mathcal{H}_{n}}(s, x, y)=\frac{1}{2^{n} n !} \sum_{\sigma \in S_{n}\left[S_{2}\right]} \operatorname{Tr}_{V}^{\otimes 2 n}\left(\sigma M^{\otimes 2 n}\right)
$$

where $M$ is again given by $s^{\Delta} x^{J_{3}^{L}} y^{J_{3}^{R}}$. This is equal to

$$
\chi_{\mathcal{H}_{n}}(s, x, y)=\sum_{p \vdash 2 n} Z_{p}^{S_{n}\left[S_{2}\right]} \prod_{i=1}\left(\operatorname{Tr} M^{i}\right)^{p_{i}}=\sum_{p \vdash 2 n} Z_{p}^{S_{n}\left[S_{2}\right]} \prod_{i=1}\left(\sum_{a} m_{a}^{i}\right)^{p_{i}}
$$

where $m_{a}$ are the eigenvalues of $M$ and $Z_{p}^{S_{n}\left[S_{2}\right]}$ is the cycle index, which gives the number of permutations in $S_{n}\left[S_{2}\right]$ with cycle structure specified by $p_{i}$. The generating function for these cycle indices is known (see e.g. [35]) and can be used to find the following generating 
function for the characters

$$
\begin{aligned}
\sum_{n=0}^{\infty} t^{n} \operatorname{Tr}_{\mathcal{H}_{n}}(M) & =\prod_{a} \frac{1}{\sqrt{1-t m_{a}^{2}}} \prod_{a \neq b} \frac{1}{\sqrt{\left(1-t m_{a} m_{b}\right)}} \\
& =\prod_{a} \frac{1}{\sqrt{1-t m_{a}^{2}}} \prod_{a>b} \frac{1}{\left(1-t m_{a} m_{b}\right)}
\end{aligned}
$$

We can now argue as we did in section 3. Using the known eigenvalues of $M$, the generalization of (3.7) is given by

$$
\begin{aligned}
\mathcal{Z}(s, x, y)= & \sum_{n=0}^{\infty} t^{n} \chi_{\mathcal{H}_{n}}(s, x, y) \\
= & \prod_{q=0}^{\infty} \prod_{a=-\frac{q}{2}}^{\frac{q}{2}} \prod_{b=-\frac{q}{2}}^{\frac{q}{2}} \frac{1}{\sqrt{1-t s^{2 q+2} x^{2 a} y^{2 b}}} \\
& \times \prod_{q_{2}=0}^{\infty} \prod_{a_{2}=-\frac{q_{2}}{2}}^{\frac{q_{2}}{2}} \prod_{b_{2}=-\frac{q_{2}}{2}}^{\frac{q_{2}}{2}} \prod_{\left(q_{1}, a_{1}, b_{1}\right)<\left(q_{2}, a_{2}, b_{2}\right)} \frac{1}{\left(1-t s^{q_{1}+q_{2}+2} x^{a_{1}+a_{2}} y^{b_{1}+b_{2}}\right)}
\end{aligned}
$$

This can be simplified further. We can order the triples $(q, a, b)$ as follows: the inequality $\left(q_{1}, a_{1}, b_{1}\right)<\left(q_{2}, a_{2}, b_{2}\right)$ means: $q_{1}<q_{2}$ or $q_{1}=q_{2}, a_{1}<a_{2}$, or $q_{1}=q_{2}, a_{1}=a_{2}, b_{1}<b_{2}$. Alternatively, we can write

$$
\begin{aligned}
\mathcal{Z}(s, x, y)= & \prod_{q=0}^{\infty} \prod_{a=-\frac{q}{2}}^{\frac{q}{2}} \prod_{b=-\frac{q}{2}}^{\frac{q}{2}} \frac{1}{\sqrt{\left(1-t s^{2 q+2} x^{2 a} y^{2 b}\right)}} \\
& \times \prod_{q_{2}=0}^{\infty} \prod_{a_{2}=-\frac{q_{2}}{2}}^{\frac{q_{2}}{2}} \prod_{b_{2}=-\frac{q_{2}}{2}}^{\frac{q_{2}}{2}} \prod_{q_{1}=0}^{\infty} \prod_{a_{1}=-\frac{q_{1}}{2}}^{\frac{q_{1}}{2}} \prod_{b_{1}=-\frac{q_{1}}{2}}^{\frac{q_{1}}{2}} \frac{1}{\sqrt{\left(1-t s^{q_{1}+q_{2}+2} x^{a_{1}+a_{2}} y^{\left.b_{1}+b_{2}\right)}\right.}}
\end{aligned}
$$

We can now define the generating function (here we take $n>1$ to avoid complications with null states)

$$
G_{2 n}^{\mathrm{O}(N)}(s, x, y)=\sum_{d=0}^{\infty} \sum_{j_{1}, j_{2}} N_{\left[2 n+d, j_{1}, j_{2}\right]}^{\mathrm{O}(N)} s^{2 n+d} x^{j_{1}} y^{j_{2}}
$$

which is given by

$$
G_{2 n}^{\mathrm{O}(N)}(s, x, y)=\left[\left(1-\frac{1}{x}\right)\left(1-\frac{1}{y}\right) Z_{2 n}(s, x, y)\right]_{\geq}
$$

where

$$
\begin{aligned}
Z_{2 n}(s, x, y) & =\chi_{\mathcal{H}_{n}}(s, x, y)(1-s \sqrt{x y})\left(1-s \sqrt{\frac{x}{y}}\right)\left(1-s \sqrt{\frac{y}{x}}\right)\left(1-\frac{s}{\sqrt{x y}}\right) \\
& =\sum_{d=0}^{\infty} \sum_{j_{1}, j_{2}} N_{\left[2 n+d, j_{1}, j_{2}\right]}^{\mathrm{O}(N)} s^{2 n+d} \chi_{j_{1}}(x) \chi_{j_{2}}(y)
\end{aligned}
$$


For $n=1$ we need to subtract out the null states that are present since the primaries being counted include conserved higher spin currents.

We can again specialize to the counting of extremal primaries. For example, the leading twist primaries are counted by $G_{2 n}^{\mathrm{O}(N), \max }(s, x, y)$ where

$$
\begin{aligned}
& \sum_{n=0}^{\infty} t^{n} G_{2 n}^{\mathrm{O}(N), \max }(s, x, y)=\sum_{n=0}^{\infty} t^{n}(1-s \sqrt{x y}) \chi_{2 n}^{\mathrm{O}(N), \max }(s, x, y) \\
& \sum_{n=0}^{\infty} \chi_{2 n}^{\mathrm{O}(N), \max }(s, x, y) t^{n}=\prod_{q=0}^{\infty} \frac{1}{\sqrt{1-t s^{2 q+2} x^{q} y^{q}}} \prod_{q_{1}, q_{2}=0}^{\infty} \frac{1}{\sqrt{1-t s^{q_{1}+q_{2}+2} x^{\frac{q_{1}+q_{2}}{2}} y^{\frac{q_{1}+q_{2}}{2}}}}
\end{aligned}
$$

It is now straightforward to obtain the Hilbert series for leading twist primaries built using 4 fields

$$
G_{4}^{\mathrm{O}(N), \max }(s, x, y)=\frac{s^{4}\left(1-s^{6} x^{3} y^{3}\right)}{\left(1-s^{2} x y\right)^{2}\left(1-s^{3} x^{\frac{3}{2}} y^{\frac{3}{2}}\right)\left(1-s^{4} x^{2} y^{2}\right)}
$$

This shows that there are 4 generators and a single relation, that this space of operators is a complete intersection and it is 3 dimensional. In a similar way we have

$$
G_{6}^{\mathrm{O}(N), \max }(s, x, y)=\frac{s^{6}\left(1-s \sqrt{x y}+s^{3} x^{\frac{3}{2}} y^{\frac{3}{2}}-s^{7} x^{\frac{7}{2}} y^{\frac{7}{2}}+s^{9} x^{\frac{9}{2}} y^{\frac{9}{2}}-s^{10} x^{5} y^{5}\right)}{(1-s \sqrt{x y})\left(1-s^{2} x y\right)^{2}\left(1-s^{3} x^{\frac{3}{2}} y^{\frac{3}{2}}\right)\left(1-s^{4} x^{2} y^{2}\right)\left(1-s^{6} x^{3} y^{3}\right)}(5
$$

The Hilbert series for these primaries are again palindromic. For the case of one-complex variable that we are discussing, we have

$$
G_{2 n}^{\mathrm{O}(N), \max }(q)=s^{2 n} \sum_{\substack{\Lambda \vdash 2 n \\ \text { Neven }}} Z_{\mathrm{SH}}(q, \Lambda)
$$

Using this formula and (4.56) we find

$$
\begin{aligned}
G_{2 n}^{\mathrm{O}(N), \max }\left(q^{-1}\right) & =s^{2 n} \sum_{\substack{\Lambda \vdash 2 n \\
\text { Neven }}} Z_{\mathrm{SH}}\left(q^{-1}, \Lambda\right) \\
& =-q^{2 n-1} s^{2 n} \sum_{\substack{\Lambda \vdash 2 n \\
\text { Neven }}} Z_{\mathrm{SH}}\left(q, \Lambda^{T}\right) \\
& =-(q)^{2 n-1} G^{\mathrm{O}(N), \max }(q)
\end{aligned}
$$

This demonstrates the palindromy property for the Hilbert series associated to the orbifold

$$
(\mathbb{C})^{2 n} /\left(\mathbb{C} \times S_{n}\left[S_{2}\right]\right)
$$

Now consider the two complex variable case.

$$
\begin{aligned}
\mathcal{Z}(s, x, y) & =\prod_{q=0}^{\infty} \frac{1}{\sqrt{1-t s^{2 q+2} x^{q} y^{q}}} \prod_{q_{1}, q_{2}=0}^{\infty} \frac{1}{\sqrt{1-t s^{q_{1}+q_{2}+2} x^{\frac{q_{1}+q_{2}}{2}} y^{\frac{q_{1}+q_{2}}{2}}}} \\
& =\sum_{t=0}^{\infty} t^{n} \chi_{\mathcal{H}_{n}, w}^{z,}(s, x, y)
\end{aligned}
$$


it is natural to consider the generating functions

$$
Z_{n}^{\mathrm{O}(N), z w}(s, x, y)=(1-s \sqrt{x y})\left(1-s \sqrt{\frac{x}{y}}\right) \chi_{\mathcal{H}_{n}}^{z, w}(s, x, y)
$$

and

$$
G_{n}^{\mathrm{O}(N), z w}=\left[\left(1-\frac{1}{y}\right) Z_{n}^{\mathrm{O}(N), z w}(s, x, y)\right]_{\geq}
$$

A straightforward computation gives

$$
Z_{4}^{\mathrm{O}(N), z w}(s, x, y)=\frac{g(s, x, y)}{\left(1-s \sqrt{\frac{x}{y}}\right)^{4}(1-s \sqrt{x y})^{4}\left(1+s \sqrt{\frac{x}{y}}\right)^{2}(1+s \sqrt{x y})^{2}\left(1+s^{2} \frac{x}{y}\right)\left(1+s^{2} x y\right)}
$$

where

$$
\begin{aligned}
g(s, x, y)= & s^{4}\left(1-\left(s \sqrt{x}+s^{3} x^{\frac{3}{2}}+s^{5} x^{\frac{5}{7}}+s^{7} x^{\frac{7}{2}}\right)\left(\sqrt{y}+\frac{1}{\sqrt{y}}\right)\right. \\
& \left.+\left(s^{8} x^{4}+s^{4} x^{2}+2 s^{2} x+2 s^{6} x^{3}\right)+\left(s^{4} x^{2}+s^{2} x+s^{6} x^{3}\right)\left(y+1+\frac{1}{y}\right)\right)
\end{aligned}
$$

This result can be recovered by using the generating function

$$
s^{4} \sum_{\Lambda_{1}, \Lambda_{2}}\left(C\left(\text { m }, \Lambda_{1}, \Lambda_{2}\right)+C\left(\boxplus, \Lambda_{1}, \Lambda_{2}\right)\right) Z_{\mathrm{SH}}\left(\Lambda_{1}, s \sqrt{x y}\right) Z_{\mathrm{SH}}\left(\Lambda_{2}, s \sqrt{\frac{x}{y}}\right)
$$

Recall that

$$
Z_{\mathrm{SH}}(\Lambda, q)=(1-q) q^{\sum_{i} c_{i}\left(c_{i}-1\right) / 2} \prod_{b} \frac{1}{\left(1-q^{h_{b}}\right)}
$$

Formula (5.22) is a consequence of the fact that an irrep $\Lambda$ of $S_{2 n}$ contains the trivial of $S_{n}\left[S_{2}\right]$ with multiplicity 1 . For the example given above, using the fact that the non-zero terms are

$$
\begin{aligned}
& C\left(\text { 巴 }, \Lambda_{1}, \Lambda_{2}\right)=\delta_{\Lambda_{1}, \Lambda_{2}} \\
& C(\text { 皿, }, \boxplus, \boxplus)=C(\boxplus, \boxplus, \boxplus)=1 \\
& C(\boxplus, \boxplus, \boxplus)=C(\boxplus, \boxplus, \boxplus)=1
\end{aligned}
$$

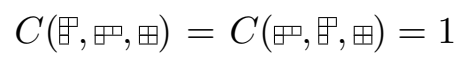

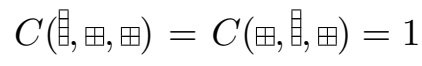

$$
\begin{aligned}
& C(\boxplus, \boxplus, \boxplus)=1
\end{aligned}
$$

we obtain complete agreement between (5.20) and (5.22). The geometries associated to $Z_{2 n}^{\mathrm{O}(N), z w}(s, x, y)$ are

$$
\frac{\left(\mathbb{C}^{2}\right)^{2 n}}{\left(\mathbb{C}^{2} \times S_{n}\left[S_{2}\right]\right)}
$$


and, after we impose the $G_{+}$condition, the geometries for $G_{2 n}^{\mathrm{O}(N), \mathrm{zw}}(s, x, y)$ are

$$
\frac{\left(\mathbb{C}^{2}\right)^{2 n}}{\left(G_{+} \times S_{n}\left[S_{2}\right]\right)}
$$

$G_{+}$is the unipotent group of upper triangular $2 \times 2$ matrices with 1 on the diagonal. For the 2-complex variables case, we have the Hilbert series

$$
Z_{n}^{\mathrm{O}(N), z w}\left(q_{1}, q_{2}\right)=s^{2 n} \sum_{\lambda_{1}, \Lambda_{2} \vdash 2 n} \sum_{\substack{\Lambda \vdash 2 n \\ \Lambda \text { even }}} C\left(\Lambda_{1}, \Lambda_{2}, \Lambda\right) Z_{\mathrm{SH}}\left(q_{1}, \Lambda_{1}\right) Z_{\mathrm{SH}}\left(q_{2}, \Lambda_{2}\right)
$$

where $C(R, S, T)$ is the Kronecker coefficient giving the number of $S_{n}$ invariants in the tensor product of three irreps $R, S, T$ of $S_{n}$. Applying the inversion

$$
\begin{aligned}
Z_{2 n}^{\mathrm{O}(N), z w}\left(q_{1}^{-1}, q_{2}^{-1}\right) & =s^{2 n} \sum_{\lambda_{1}, \Lambda_{2} \vdash 2 n} \sum_{\substack{\Lambda \vdash 2 n \\
\Lambda \text { even }}} C\left(\Lambda_{1}, \Lambda_{2}, \Lambda\right) Z_{\mathrm{SH}}\left(q_{1}^{-1}, \Lambda_{1}\right) Z_{\mathrm{SH}}\left(q_{2}^{-1}, \Lambda_{2}\right) \\
& =s^{2 n}\left(q_{1} q_{2}\right)^{2 n-1} \sum_{\lambda_{1}, \Lambda_{2} \vdash 2 n} \sum_{\substack{\Lambda \vdash 2 n \\
\Lambda \text { even }}} C\left(\Lambda_{1}, \Lambda_{2}, \Lambda\right) Z_{\mathrm{SH}}\left(q_{1}, \Lambda_{1}^{T}\right) Z_{\mathrm{SH}}\left(q_{2}, \Lambda_{2}^{T}\right) \\
& =s^{2 n}\left(q_{1} q_{2}\right)^{2 n-2} \sum_{\lambda_{1}, \Lambda_{2} \vdash 2 n} \sum_{\substack{\Lambda \vdash 2 n \\
\Lambda \text { even }}} C\left(\Lambda_{1}^{T}, \Lambda_{2}^{T}, \Lambda\right) Z_{\mathrm{SH}}\left(q_{1}, \Lambda_{1}\right) Z_{\mathrm{SH}}\left(q_{2}, \Lambda_{2}\right) \\
& =s^{2 n}\left(q_{1} q_{2}\right)^{2 n-1} \sum_{\lambda_{1}, \Lambda_{2} \vdash 2 n} \sum_{\substack{\Lambda \vdash 2 n \\
\Lambda \text { even }}} C\left(\Lambda_{1}, \Lambda_{2}, \Lambda\right) Z_{\mathrm{SH}}\left(q_{1}, \Lambda_{1}\right) Z_{\mathrm{SH}}\left(q_{2}, \Lambda_{2}\right) \\
& =\left(q_{1} q_{2}\right)^{2 n-1} Z_{n}^{\mathrm{O}(N), z w}\left(q_{1}, q_{2}\right)
\end{aligned}
$$

In going from the second to third line, we renamed $\Lambda_{1} \rightarrow \Lambda_{1}^{T}, \Lambda_{2} \rightarrow \Lambda_{2}^{T}$. In going from the third to fourth line, we used an invariance of the Kronecker multiplicity

$$
C\left(\Lambda_{1}, \Lambda_{2}, \Lambda\right)=C\left(\Lambda_{1}^{T}, \Lambda_{2}^{T}, \Lambda\right)
$$

which follows from

$$
C\left(\Lambda_{1}, \Lambda_{2}, \Lambda\right)=\frac{1}{(2 n) !} \sum_{\sigma \in S_{2 n}} \chi_{\Lambda_{1}}(\sigma) \chi_{\Lambda_{2}}(\sigma) \chi_{\Lambda}(\sigma)
$$

and

$$
\chi_{\Lambda^{T}}(\sigma)=(-1)^{\sigma} \chi_{\Lambda}(\sigma)
$$

where $(-1)^{\sigma}$ is the parity of $\sigma$. The formula (5.28) demonstrates that the palindromy property of the Hilbert series for the counting of vector model primaries.

\section{$6 \quad$ Matrix model primaries}

Another interesting generalization of the single real scalar field, is to a matrix scalar. We gauge the free theory. The net effect is that we look for primary operators with all indices contracted. There are many ways that the indices can be contracted, corresponding to the 
different possible multitrace structures that can be written down. Thus, generalizing to the matrix scalar introduces an interesting non-trivial structure to the problem.

The large $N$ counting of gauge invariant functions of a single matrix, is achieved by integrating [32]

$$
\mathcal{Z}(x)=\int d U e^{\sum_{i} \frac{x^{i}}{i}(\operatorname{tr} U)^{i} \operatorname{tr}\left(U^{\dagger}\right)^{i}}=\prod_{i=1}^{\infty} \frac{1}{\left(1-x^{i}\right)}
$$

For multi-matrices, the large $N$ counting is [32]

$$
\mathcal{Z}\left(x_{i}\right)=\int d U e^{\sum_{i} \frac{\left(\sum_{a} x_{a}^{i}\right)}{i}(\operatorname{tr} U)^{i} \operatorname{tr}\left(U^{\dagger}\right)^{i}}=\prod_{i=1}^{\infty} \frac{1}{\left(1-\sum_{a=1}^{M} x_{a}^{i}\right)}
$$

where $M$ is the number of matrices in the model. Specializing to the 2-matrix case, this is

$$
\mathcal{Z}(x, y)=\prod_{i=1}^{\infty} \frac{1}{\left(1-x^{i}-y^{i}\right)}
$$

For the matrix scalar, we have matrix fields

$$
\partial_{l, m} \phi_{j}^{i}
$$

$l$ denotes a symmetric traceless irrep of $\mathrm{SO}(4)$ and $m$ runs over the states in this irrep. There are known methods that can be used to write diagonal bases for the local operators of this theory $[15,33]$. For the large $N$ counting of gauge invariants built from derivatives of a single matrix, we have [34]

$$
\mathcal{Z}(t, s, x, y)=\int d U e^{\sum_{i=1}^{\infty} \sum_{q=0}^{\infty} \sum_{a_{q}, b_{q}=-\frac{q}{2}}^{\frac{q}{2}} \frac{\left(t_{s}(1+q) x^{a} y_{y} b_{q}\right)^{i}}{i} \operatorname{tr} U^{i} \operatorname{tr}\left(U^{\dagger}\right)^{i}}
$$

Note that this can also be written as

$$
\mathcal{Z}(t, s, x, y)=\int d U e^{\sum_{i=1}^{\infty} \frac{t^{i}}{i} \chi_{V^{+}}\left(s^{i}, x^{i}, y^{i}\right) \operatorname{tr} U^{i} \operatorname{tr}\left(U^{\dagger}\right)^{i}}
$$

By repeating steps similar to the ones we did for the integral encountered in case of multimatrices, we get

$$
\mathcal{Z}(t, s, x, y)=\prod_{i=1}^{\infty} \frac{1}{\left(1-\sum_{q=0}^{\infty} \sum_{a_{q}, b_{q}=-\frac{q}{2}}^{\frac{q}{2}} t^{i} s^{i+q i} x^{i a_{q}} y^{i b_{q}}\right)}
$$

To simplify this further, we will derive an identity quoted in [32]. The state space of a single scalar $V_{+}$is obtained by acting on the ground state with products of the operators $P_{\mu}$. This is a $4 \mathrm{D}$ irrep of $\mathrm{SO}(4)=\mathrm{SU}(2) \times \mathrm{SU}(2)$ with spins $(1 / 2,1 / 2)$. The equation of motion says that $P_{\mu} P_{\mu}$ acting on the ground state is zero. An immediate consequence is that the independent states in $V_{+}$generated by $q$ copies of $P$ transform as the symmetric 
traceless irrep of $\mathrm{SO}(4)$, corresponding to the Young diagram with a single row of length $q$. This irrep of $\mathrm{SO}(4)$ is the $(q / 2, q / 2)$ irrep of $\mathrm{SU}(2) \times \mathrm{SU}(2)$. It immediately follows that

$$
\begin{aligned}
\chi_{V_{+}}(s, x, y) & =\operatorname{tr}_{V_{+}}\left(s^{D} x^{J_{L}} y^{J_{R}}\right) \\
& =s \sum_{q=0}^{\infty} s^{q} \chi_{q / 2}(x) \chi_{q / 2}(y) \\
& =s \sum_{q=0}^{\infty} s^{q} \sum_{a_{q}=-q / 2}^{q / 2} x^{a_{q}} \sum_{b_{q}=-q / 2}^{q / 2} y^{b_{q}}
\end{aligned}
$$

This character was used above in (6.5) and (6.6). The state space obtained by acting with all the $P_{\mu}$ 's, without setting $P_{\mu} P_{\mu}=0$ has character

$$
\chi_{\tilde{V}_{+}}(s, x, y)=t r_{\tilde{V}_{+}} s^{D} x^{J_{L}} y^{J_{R}}=s \sum_{p=0}^{\infty} \sum_{q=0}^{\infty} s^{2 p} s^{q} \chi_{q / 2}(x) \chi_{q / 2}(y)
$$

The $p$ summation is over the number of powers of $P^{2}$. A basis in $\tilde{V}_{+}$can be given by multiplying powers of $P^{2}$ with traceless products. Doing the sum over $p$, we find

$$
\chi_{\widetilde{V}_{+}}(s, x, y)=s\left(1-s^{2}\right)^{-1} \chi_{V^{+}}(s, x, y)
$$

so that

$$
\chi_{V^{+}}(s, x, y)=\left(1-s^{2}\right) s^{-1} \chi_{\widetilde{V}_{+}}(s, x, y)
$$

Now by thinking about $\widetilde{V}_{+}$as isomorphic to the Fock space generated by four oscillators $P_{\mu}$ (which transform in the $(1 / 2,1 / 2)$ of $\left.\mathrm{SU}(2) \times \mathrm{SU}(2)\right)$ it is evident that

$$
\chi_{\widetilde{V}_{+}}(s, x, y)=\frac{s}{(1-s \sqrt{x y})\left(1-s \sqrt{\frac{x}{y}}\right)(1-s / \sqrt{x y})\left(1-s \sqrt{\frac{y}{x}}\right)} \equiv s P(s, x, y)
$$

and so we find

$$
\chi_{V^{+}}(s, x, y)=\left(1-s^{2}\right) P(s, x, y)=s \sum_{q=0}^{\infty} s^{q} \sum_{a_{q}=-q / 2}^{q / 2} x^{a_{q}} \sum_{b_{q}=-q / 2}^{q / 2} y^{b_{q}}
$$

Thus, we have the identity

$$
\sum_{q=0}^{\infty} s^{q} \sum_{a_{q}=-q / 2}^{q / 2} x^{a_{q}} \sum_{b_{q}=-q / 2}^{q / 2} y^{b_{q}}=\frac{-\left(s-s^{-1}\right)}{(1-s \sqrt{x y})\left(1-s \sqrt{\frac{x}{y}}\right)(1-s / \sqrt{x y})\left(1-s \sqrt{\frac{y}{x}}\right)}
$$

Using this identity, we can now rewrite (6.7) as

$$
\begin{aligned}
\mathcal{Z}(s, x, y) & =\prod_{i=1}^{\infty}\left(1+\frac{(t s)^{i}\left(s^{i}-s^{-i}\right)}{\left(1-s^{i} \sqrt{x^{i} y^{i}}\right)\left(1-s^{i} \sqrt{\frac{x^{i}}{y^{i}}}\right)\left(1-s^{i} \sqrt{\frac{y^{i}}{x^{i}}}\right)\left(1-s^{i} / \sqrt{x^{i} y^{i}}\right)}\right)^{-1} \\
& =\sum_{n=0}^{\infty} t^{n} \chi_{n}(s, x, y)
\end{aligned}
$$


As we did above, we can define two primary generating functions as follows

$$
\begin{aligned}
Z_{n}(s, x, y) & =\sum_{\Delta} \sum_{j_{1}, j_{2}} \mathcal{N}_{\left[\Delta, j_{1}, j_{2}\right]}^{(n)} s^{\Delta} \chi_{j_{1}}(x) \chi_{j_{2}}(y) \\
& =\chi_{n}(s, x, y)(1-s \sqrt{x y})\left(1-s \sqrt{\frac{x}{y}}\right)\left(1-s \sqrt{\frac{y}{x}}\right)\left(1-\frac{s}{\sqrt{x y}}\right)
\end{aligned}
$$

and

$$
\begin{aligned}
G_{n}(s, x, y) & =\sum_{\Delta} \sum_{j_{1}, j_{2}} \mathcal{N}_{\left[\Delta, j_{1}, j_{2}\right]}^{(n)} s^{\Delta} x^{j_{1}} y^{j_{2}} \\
& =\left[\left(1-\frac{1}{x}\right)\left(1-\frac{1}{y}\right) Z_{n}(s, x, y)\right]_{\geq}
\end{aligned}
$$

Here $\mathcal{N}_{\left[\Delta, j_{1}, j_{2}\right]}^{(n)}$ counts the number of primaries of dimension $\Delta$ and spins $\left(j_{1}, j_{2}\right)$ that can be constructed using $n$ matrix fields. We can again specialize the counting to counting leading twist primaries, or to count extremal primaries. The relevant generating function for the counting of extremal primaries is given by

$$
Z_{n}^{z w}(s, x, y)=s^{n} \sum_{\Lambda_{1}, \Lambda_{2} \vdash n} \sum_{R \Lambda \vdash n} Z_{\mathrm{SH}}\left(s \sqrt{x y}, \Lambda_{1}\right) Z_{\mathrm{SH}}\left(s \sqrt{\frac{x}{y}}, \Lambda_{2}\right) C\left(\Lambda_{1}, \Lambda_{2}, \Lambda\right) C(R, R, \Lambda)
$$

This follows from the general counting of matrix gauge invariants in the case where the matrices $X_{a}$ transform under some global symmetry group $G$, given in [15]. The resulting Hilbert series, for $n=3$, is

$$
\begin{aligned}
Z_{3}^{z w}= & \frac{s^{3} \Upsilon(s, x, y)}{\left(1-s \sqrt{\frac{x}{y}}\right)^{2}\left(1+s \sqrt{\frac{x}{y}}\right)(-1+s \sqrt{x y})^{2}(1+s \sqrt{x y})\left(s^{2} \frac{x}{y}+s \sqrt{\frac{x}{y}}+1\right)\left(1+s \sqrt{x y}+s^{2} x y\right)} \\
\Upsilon(s, x, y)= & 3+3 s^{6} x^{3}+\left(s \sqrt{x}+s^{5} x^{\frac{5}{2}}\right)\left(\frac{1}{\sqrt{y}}+\sqrt{y}\right)+\left(s^{2} x+s^{4} x^{2}\right)\left(\frac{1}{y}+5+y\right) \\
& +s^{3} x^{\frac{3}{2}}\left(\frac{1}{y^{\frac{3}{2}}}+\frac{5}{\sqrt{y}}+5 \sqrt{y}+y^{\frac{3}{2}}\right)
\end{aligned}
$$

This counts the total number of primaries we can build from 3 matrix fields. We can refine this counting by specifying the trace structure. Schematically, the primaries we study have the form

$$
\mathcal{O}=\left.\sum_{\vec{n}, \vec{m}} c_{\vec{n} \vec{m}} \partial_{z_{1}}^{n_{1}} \partial_{w_{1}}^{m_{1}} \phi_{i_{\sigma(1)}}^{i_{1}} \partial_{z_{2}}^{n_{2}} \partial_{w_{2}}^{m_{2}} \phi_{i_{\sigma(2)}}^{i_{2}} \partial_{z_{3}}^{n_{3}} \partial_{w_{3}}^{m_{3}} \phi_{i_{\sigma(3)}}^{i_{3}}\right|_{z_{k}=z, w_{k}=w}
$$

i.e. they are specified by allowing derivatives to act on some gauge invariant operator specified by the permutation $\sigma \in S_{n}$. After we translate to the polynomial language, primaries are specified by polynomials in $n$ variables $z_{i}$ and $w_{i}$, as well as by the trace structure, i.e. they are functions on the space

$$
\frac{\left(\mathbb{C}^{2}\right)^{n}}{\mathbb{C}^{2}} \times S_{n}
$$


These functions have to be invariant under an action of $\gamma \in S_{n}$

$$
\gamma:\left(w_{I}, z_{J}, \sigma\right) \rightarrow\left(w_{\gamma(I)}, z_{\gamma(I)}, \gamma^{-1} \sigma \gamma\right) \quad \gamma \in S_{n}
$$

Modding out by this symmetry we find the primaries are functions on the space

$$
\frac{\left(\left(\mathbb{C}^{2}\right)^{n} \times S_{n}\right)}{\left(\mathbb{C}^{2} \times S_{n}\right)}
$$

We can also obtain a description by fixing a specific permutation, and then dividing by those permutations $\gamma$ that fix $\sigma$. Lets work out this description for $n=3$. For primaries obtained by acting with derivatives on $\operatorname{Tr}(\phi)^{3}, \sigma=(1)(2)(3)$ which is left invariant by $\gamma \in S_{3}$. Thus, we need to consider

$$
\frac{\left(\mathbb{C}^{2}\right)^{3}}{\left(\mathbb{C}^{2} \times S_{3}\right)}
$$

We need to project to the trivial of $S_{3}$ and hence

$$
\begin{aligned}
Z_{(\operatorname{Tr} \phi)^{3}}^{z w} & =s^{3} \sum_{\Lambda \vdash 3} Z_{\mathrm{SH}}(s \sqrt{x y}, \Lambda) Z_{\mathrm{SH}}\left(s \sqrt{\frac{x}{y}}, \Lambda\right) \\
& =\frac{s^{3}\left(1+s^{2} x+s^{4} x^{2}+s^{6} x^{3}+s^{3} x^{\frac{3}{2}}\left(\frac{1}{\sqrt{y}}+\sqrt{y}\right)\right)}{(1-s \sqrt{x y})^{2}(1+s \sqrt{x y})(-1+s \sqrt{x y})^{2}(1+s \sqrt{x y})\left(s^{2} \frac{x}{y}+s \sqrt{\frac{x}{y}}+1\right)\left(1+s \sqrt{x y}+s^{2} x y\right)}
\end{aligned}
$$

For primaries obtained by acting with derivatives on $\left(\operatorname{Tr} \phi^{2}\right) \operatorname{Tr}(\phi)$, we can choose $\sigma=$ (12)(3) which is left invariant by $S_{2} \times S_{1}$. Thus, we need to consider

$$
\frac{\left(\mathbb{C}^{2}\right)^{3}}{\left(\mathbb{C}^{2} \times S_{2} \times S_{1}\right)}
$$

where $S_{2}$ contains permutations of $\left(z_{1}, w_{1}\right)$ and $\left(z_{2}, w_{2}\right)$. Thus, we need to project to the trivial (๓, ) of the $S_{2} \times S_{1}$ subgroup. This representation is subduced once by $\mathrm{m}$ and once by $\bullet$. Thus

$$
\begin{aligned}
Z_{\left(\operatorname{Tr} \phi^{2}\right) \operatorname{Tr}(\phi)}^{z w}= & s^{3} Z_{\mathrm{SH}}(s \sqrt{x y}, \square) Z_{\mathrm{SH}}\left(s \sqrt{\frac{x}{y}}, \square\right)+2 s^{3} Z_{\mathrm{SH}}(s \sqrt{x y}, \boxminus) Z_{\mathrm{SH}}\left(s \sqrt{\frac{x}{y}}, \square\right) \\
& +s^{3} Z_{\mathrm{SH}}(s \sqrt{x y}, \boxminus) Z_{\mathrm{SH}}\left(s \sqrt{\frac{x}{y}}, \boxminus\right)+s^{3} Z_{\mathrm{SH}}(s \sqrt{x y}, \square) Z_{\mathrm{SH}}\left(s \sqrt{\frac{x}{y}}, \square\right) \\
& +s^{3} Z_{\mathrm{SH}}(s \sqrt{x y}, \boxminus) Z_{\mathrm{SH}}\left(s \sqrt{\frac{x}{y}}, \square\right)+s^{3} Z_{\mathrm{SH}}(s \sqrt{x y}, \square) Z_{\mathrm{SH}}\left(s \sqrt{\frac{x}{y}}, \boxminus\right) \\
& +s^{3} Z_{\mathrm{SH}}(s \sqrt{x y}, \boxminus) Z_{\mathrm{SH}}\left(s \sqrt{\frac{x}{y}}, \square\right) \\
= & \frac{s^{3}\left(1+s^{2} x\right)}{(1-s \sqrt{x y})^{2}(1+s \sqrt{x y})(-1+s \sqrt{x y})^{2}(1+s \sqrt{x y})}
\end{aligned}
$$

For primaries obtained by acting with derivatives on $\operatorname{Tr}\left(\phi^{3}\right)$, we can take $\sigma=(123)$ which is left invariant by $Z_{3}$. Thus, need to consider

$$
\frac{\left(\mathbb{C}^{2}\right)^{3}}{\left(\mathbb{C}^{2} \times Z_{3}\right)}
$$


where $Z_{3}$ is the group comprising $\{1,(123),(132)\}$. We need to project to the trivial of $Z_{3}$. The trivial of $Z_{3}$ is subduced once by $\square$ and once by $\boxminus$. Thus

$$
\begin{aligned}
Z_{\left(\operatorname{Tr} \phi^{3}\right)}^{z w}= & s^{3} Z_{\mathrm{SH}}(s \sqrt{x y}, \square) Z_{\mathrm{SH}}\left(s \sqrt{\frac{x}{y}}, \square\right)+2 s^{3} Z_{\mathrm{SH}}(s \sqrt{x y}, \square) Z_{\mathrm{SH}}\left(s \sqrt{\frac{x}{y}}, \boxminus\right) \\
& +s^{3} Z_{\mathrm{SH}}(s \sqrt{x y}, \boxminus) Z_{\mathrm{SH}}\left(s \sqrt{\frac{x}{y}}, \boxminus\right)+s^{3} Z_{\mathrm{SH}}(s \sqrt{x y}, \square) Z_{\mathrm{SH}}\left(s \sqrt{\frac{x}{y}}, \boxminus\right) \\
& +s^{3} Z_{\mathrm{SH}}(s \sqrt{x y}, \boxminus) Z_{\mathrm{SH}}\left(s \sqrt{\frac{x}{y}}, \square\right) \\
= & \frac{s^{3}\left(1+s^{4} x^{2}-\left(s \sqrt{x}+s^{3} x^{\frac{3}{2}}\right)\left(\frac{1}{\sqrt{y}}+\sqrt{y}\right)+s^{2} x\left(\frac{1}{y}+3+y\right)\right)}{\left(1-s \sqrt{\frac{x}{y}}\right)^{2}(1-s \sqrt{x y})^{2}\left(s^{2} \frac{x}{y}+s \sqrt{\frac{x}{y}}+1\right)\left(1+s \sqrt{x y}+s^{2} x y\right)}
\end{aligned}
$$

Note that

$$
Z_{3}^{z w}=Z_{\operatorname{Tr}(\phi)^{3}}^{z w}+Z_{\operatorname{Tr}\left(\phi^{2}\right) \operatorname{Tr}(\phi)}^{z w}+Z_{\operatorname{Tr}\left(\phi^{3}\right)}^{z w}
$$

as it must be. The permutation quotient geometry which includes all trace structures is

$$
\frac{\left(\mathbb{C}^{2}\right)^{n} \times S_{n}}{\left(\mathbb{C}^{2} \times S_{n}\right)}
$$

This has an $\mathrm{SU}(2)$ action. We can again look at functions which are annihilated by $J_{+}$. Let $G_{+}$be the subalgebra of $\operatorname{GL}(2, \mathbb{C})$ generated by $J_{+}$. The Hilbert series in this case is $G_{n}^{z w}$. The algebra of functions annihilated by $J_{+}$corresponds to functions on

$$
\frac{\left(\mathbb{C}^{2}\right)^{n} \times S_{n}}{\left(\mathbb{C}^{2} \times S_{n} \times G_{+}\right)}
$$

It is again possible to establish the palindromic property for the Hilbert series relevant for the matrix case. In the matrix case, we have the counting function

$$
Z_{n}^{z w}\left(q_{1}, q_{2}\right)=s^{n} \sum_{\Lambda_{1}, \Lambda_{2} \vdash n} \sum_{R \vdash n} C\left(\Lambda_{1}, \Lambda_{2}, \Lambda\right) C(R, R, \Lambda) Z_{\mathrm{SH}}\left(q_{1}, \Lambda_{1}\right) Z_{\mathrm{SH}}\left(q_{2}, \Lambda_{2}\right)
$$

The symmetry under $q_{1} \leftrightarrow q_{2}$, equivalently $x \rightarrow x, y \rightarrow y^{-1}$ is clear. Now apply inversion

$$
\begin{aligned}
Z_{n}^{z w}\left(q_{1}^{-1}, q_{2}^{-1}\right) & =s^{n} \sum_{\Lambda_{1}, \Lambda_{2} \vdash n} \sum_{R \vdash n} C\left(\Lambda_{1}, \Lambda_{2}, \Lambda\right) C(R, R, \Lambda) Z_{\mathrm{SH}}\left(q_{1}^{-1}, \Lambda_{1}\right) Z_{\mathrm{SH}}\left(q_{2}^{-1}, \Lambda_{2}\right) \\
& =s^{n}\left(q_{1} q_{2}\right)^{n-1} \sum_{\Lambda_{1}, \Lambda_{2} \vdash n} \sum_{R \vdash n} C\left(\Lambda_{1}, \Lambda_{2}, \Lambda\right) C(R, R, \Lambda) Z_{\mathrm{SH}}\left(q_{1}, \Lambda_{1}^{T}\right) Z_{\mathrm{SH}}\left(q_{2}, \Lambda_{2}^{T}\right) \\
& =s^{n}\left(q_{1} q_{2}\right)^{n-1} \sum_{\Lambda_{1}, \Lambda_{2} \vdash n} \sum_{R \vdash n} C\left(\Lambda_{1}^{T}, \Lambda_{2}^{T}, \Lambda\right) C(R, R, \Lambda) Z_{\mathrm{SH}}\left(q_{1}, \Lambda_{1}\right) Z_{\mathrm{SH}}\left(q_{2}, \Lambda_{2}\right) \\
& =s^{n}\left(q_{1} q_{2}\right)^{n-1} \sum_{\Lambda_{1}, \Lambda_{2} \vdash n} \sum_{R \vdash n} C\left(\Lambda_{1}, \Lambda_{2}, \Lambda\right) C(R, R, \Lambda) Z_{\mathrm{SH}}\left(q_{1}, \Lambda_{1}\right) Z_{\mathrm{SH}}\left(q_{2}, \Lambda_{2}\right) \\
& =\left(q_{1} q_{2}\right)^{n-1} Z_{n}^{z w}\left(q_{1}, q_{2}\right)
\end{aligned}
$$




\section{Summary and outlook}

We mapped the algebraic problem of constructing primary fields in the quantum field theory of a free scalar field $\phi$ in four dimensions to one of finding polynomial functions on $\left(\mathbb{R}^{4}\right)^{n}$ subject to constraints involving Laplace's equation on each factor, a condition of invariance under translations by the diagonal $\mathbb{R}^{4}$ and an $S_{n}$ symmetry related to the bosonic statistics of the elementary field (2.25). By considering holomophic solutions to the Laplacian conditions, we mapped the primary fields to functions on the complex orbifold

$$
\left(\mathbb{C}^{2}\right)^{n} /\left(\mathbb{C}^{2} \times S_{n}\right)
$$

We showed that this space has a palindromic Hilbert series and is Calabi-Yau. We generalized the discussion to the quantum field theory of free vector fields $\phi_{I}(x)$ in the large $N$ limit and found that the orbifold

$$
\left(\mathbb{C}^{2}\right)^{2 n} /\left(\mathbb{C}^{2} \times S_{n}\left[S_{2}\right]\right)
$$

plays an analogous role. We established the palindromy property. We then considered the free matrix scalar in four dimensions $\phi_{i}^{j}(x)$ again in the large $N$ limit. The orbifold is now

$$
\left(\left(\mathbb{C}^{2}\right)^{n} \times S_{n}\right) /\left(\mathbb{C}^{2} \times S_{n}\right)
$$

We established the palindromy of the Hilbert series.

In this paper we have focused on the explicit construction of extremal primary fields. However, the formulation of the problem of constructing general primary fields given in (2.25), as a system of equations for harmonic polynomal functions on $\left(\mathbb{R}^{4}\right)^{n}$, should be useful beyond the extremal sector. In this more general case, we have to include nonholomorphic solutions to the harmonic constraints - solving this simultaneously with the symmetry and translation constraints proves surprisingly tricky. In this case, we do not expect the ring structure of the extremal primaries to survive. Our preliminary investigations indicate that this most general problem has a graph-theoretic formulation, which will be interesting to exploit. At the level of counting these primaries, we still have the full expressions for the $s o(4,2)$ characters of $\operatorname{Sym}^{n}\left(V_{+}\right)$which, once expanded in terms of irreducible representations, will in principle yield the counting for the general case. However finding explicit expressions analogous to (3.46) or (3.50) looks challenging. It would very interesting to explore the possible application of the higher spin symmetries and twistor space variables of $[36,37]$ in shedding light on this problem. It is interesting to note that symmetric group representation theoretic questions close to (but not identical) to the ones we have used have played a role in the discussion of higher spin symmetries in [20]. Some recent mathematical results on these symmetric group multiplicities are in [38].

A number of immediate generalizations of the current work are: free fermions, gauge fields, the free limit of QCD and supersymmetric theories. Some of the early constructions of primary fields - in the SL(2) sector which is a special case of the extremal operators we considered - were done in the context of deep inelastic scattering in QCD (see for example the review [39]). It will be fascinating to explore QCD applications of the holomorphic 
primaries considered here. The explicit enumeration and construction of superconformal primary fields in $N=4 \mathrm{SYM}$ will give a better understanding of the dual $A d S_{5} \times S^{5}$ background. While the map between branes and geometries in the half-BPS sector of the bulk and the half-BPS states in $N=4 \mathrm{SYM}$ [40-42] is reasonably well understood, there are important open problems, most notably in the sector of sixteenth BPS states [43] but also in the quarter and eighth-BPS sectors (some progress on branes states in these sectors is in [44-51]). A better understanding of operators with derivatives is a step in the direction of a more complete picture of the duality map in general. The construction of holomorphic primaries for the 1-matrix case should admit, without much diffculty, generalization to multi-matrix systems and more generally to quiver theories by combining the methods of the present paper with those of $[15,52-57]$. Another natural direction is to consider correlators involving the extremal primary fields and the determination of anomalous dimensions for these fields at the Wilson-Fischer fixed point using the techniques of [6].

\section{Acknowledgments}

This work of RdMK, PR and RR is supported by the South African Research Chairs Initiative of the Department of Science and Technology and National Research Foundation as well as funds received from the National Institute for Theoretical Physics (NITheP). SR is supported by the STFC consolidated grant ST/L000415/1 "String Theory, Gauge Theory \& Duality" and a Visiting Professorship at the University of the Witwatersrand, funded by a Simons Foundation grant held at the Mandelstam Institute for Theoretical Physics. SR thanks the Galileo Galilei Institute for Theoretical Physics for hospitality and the INFN for partial support during the completion of this work. We are grateful for useful discussions to Alberto Cazzaniga, Danilo Diaz, Yang Hui He, Dario Martelli, Vishnu Jejjala, Bogdan Stefanski, Alessandro Torielli.

\section{A Decomposing $\operatorname{Sym}^{n}\left(V_{+}\right)$for small values of $n$}

In this appendix we will discuss an alternative approach to the problem of decomposing $\operatorname{Sym}\left(V_{+}^{\otimes n}\right)$ into irreps for some low values of $n$. This approach was developed in detail in [1]. The results obtained using the methods outlined in this appendix are in complete agreement with the results derived in section 3. The method of [1] starts with the observation that projection onto the completely symmetrized representation is easily accomplished with the help of Young projectors. For example, for $n=3$ we have

$$
\chi_{\mathrm{Sym}^{3}(V)}=\frac{1}{6}\left(\left(\chi_{V_{+}}(s, x, y)\right)^{3}+3 \chi_{V_{+}}(s, x, y) \chi_{V_{+}}\left(s^{2}, x^{2}, y^{2}\right)+2 \chi_{V_{+}}\left(s^{3}, x^{3}, y^{3}\right)\right)
$$

Evaluating the right hand side is most easily achieved by using the formula

$$
\begin{aligned}
\chi_{V^{+}}\left(s^{n}, x^{n}, y^{n}\right)= & P\left(s^{n}, x^{n}, y^{n}\right) s^{n}\left(1-s^{2 n}\right) \\
= & s^{n} \sum_{q=0}^{\infty} s^{n q}\left[\sum_{l=0,1, \ldots}^{\lfloor q / 2\rfloor} \chi_{\frac{q n}{2}-n l}(x)-\sum_{l=0,1, \ldots}^{\lfloor(q-1) / 2\rfloor} \chi_{\frac{q n}{2}-n l-1}(x)\right] \\
& \times\left[\sum_{l=0,1, \ldots}^{\lfloor q / 2\rfloor} \chi_{\frac{q n}{2}-n l}(y)-\sum_{l=0,1, \ldots}^{\lfloor(q-1) / 2\rfloor} \chi_{\frac{q n}{2}-n l-1}(y)\right]
\end{aligned}
$$


We also need an identity which rewrites $\chi_{V^{+}}\left(s^{n}, x^{n}, y^{n}\right)$ as $\mathrm{SU}(2)$ characters multiplied by $P(s, x, y)$; these can very easily be translated into $\mathcal{A}_{[\cdot,,,]} \mathrm{s}$, in the notation of [32]. This is easily achieved by using the well known product rule for $\mathrm{SU}(2)$ characters as well as the identity

$$
\begin{aligned}
1 & =P(s, x, y)\left(1-s x^{1 / 2} y^{1 / 2}\right)\left(1-s x^{1 / 2} y^{-1 / 2}\right)\left(1-s x^{-1 / 2} y^{1 / 2}\right)\left(1-s x^{-1 / 2} y^{-1 / 2}\right) \\
& =P(s, x, y)\left[1+s^{4}-s\left(1+s^{2}\right) \chi_{\frac{1}{2}}(x) \chi_{\frac{1}{2}}(y)+s^{2}\left(\chi_{1}(x)+\chi_{1}(y)\right)\right]
\end{aligned}
$$

A straight forward computation now gives the desired decomposition. A few examples of the method are given below.

$$
\begin{aligned}
\chi_{\mathrm{Sym}^{3}(V)}= & \mathcal{A}_{[3,0,0]}+\mathcal{A}_{[5,1,1]}+\mathcal{A}_{\left[6, \frac{3}{2}, \frac{3}{2}\right]}+\mathcal{A}_{[7,2,2]}+\mathcal{A}_{[7,0,2]}+\mathcal{A}_{[7,2,0]} \\
& +\mathcal{A}_{\left[8, \frac{5}{2}, \frac{5}{2}\right]}+\mathcal{A}_{\left[8, \frac{3}{2}, \frac{5}{2}\right]}+\mathcal{A}_{\left[8, \frac{5}{2}, \frac{3}{2}\right]}+2 \mathcal{A}_{[9,3,3]}+\mathcal{A}_{[9,1,3]}+\mathcal{A}_{[9,3,1]} \\
& +\mathcal{A}_{\left[10, \frac{7}{2}, \frac{7}{2}\right]}+\mathcal{A}_{\left[10, \frac{7}{2}, \frac{5}{2}\right]}+\mathcal{A}_{\left[10, \frac{5}{2}, \frac{7}{2}\right]}+\mathcal{A}_{\left[10, \frac{7}{2}, \frac{3}{2}\right]}+\mathcal{A}_{\left[10, \frac{3}{2}, \frac{7}{2}\right]}+\ldots \\
\chi_{\mathrm{Sym}^{4}(V)}= & \mathcal{A}_{[4,0,0]}+\mathcal{A}_{[6,1,1]}+\mathcal{A}_{\left[7, \frac{3}{2}, \frac{3}{2}\right]}+\mathcal{A}_{[8,0,0]}+\mathcal{A}_{[8,0,2]}+\mathcal{A}_{[8,2,0]}+\mathcal{A}_{[8,1,1]}+2 \mathcal{A}_{[8,2,2]} \\
& +\mathcal{A}_{\left[9, \frac{3}{2}, \frac{1}{2}\right]}+\mathcal{A}_{\left[9, \frac{5}{2}, \frac{1}{2}\right]}+\mathcal{A}_{\left[9, \frac{1}{2}, \frac{3}{2}\right]}+\mathcal{A}_{\left[9, \frac{5}{2}, \frac{3}{2}\right]}+\mathcal{A}_{\left[9, \frac{1}{2}, \frac{5}{2}\right]}+\mathcal{A}_{\left[9, \frac{3}{2}, \frac{5}{2}\right]}+\mathcal{A}_{\left[9, \frac{5}{2}, \frac{5}{2}\right]} \\
& +\mathcal{A}_{[10,0,0]}+2 \mathcal{A}_{[10,1,1]}+\mathcal{A}_{[10,2,1]}+2 \mathcal{A}_{[10,3,1]}+\mathcal{A}_{[10,1,2]}+2 \mathcal{A}_{[10,2,2]}+\mathcal{A}_{[10,3,2]} \\
& +2 \mathcal{A}_{[10,1,3]}+\mathcal{A}_{[10,2,3]}+3 \mathcal{A}_{[10,3,3]}+\ldots \\
\chi_{\mathrm{Sym}^{5}(V)}+\ldots & \mathcal{A}_{[5,0,0]}+\mathcal{A}_{[7,1,1]}+\mathcal{A}_{\left[8, \frac{3}{2}, \frac{3}{2}\right]}+\mathcal{A}_{[9,0,0]}+\mathcal{A}_{[9,1,1]}+\mathcal{A}_{[9,2,0]}+\mathcal{A}_{[9,0,2]}+2 \mathcal{A}_{[9,2,2]} \\
& +\mathcal{A}_{\left[10, \frac{1}{2}, \frac{1}{2}\right]}+\mathcal{A}_{\left[10, \frac{3}{2}, \frac{1}{2}\right]}+\mathcal{A}_{\left[10, \frac{1}{2}, \frac{3}{2}\right]}+\mathcal{A}_{\left[10, \frac{3}{2}, \frac{3}{2}\right]}+\mathcal{A}_{\left[10, \frac{1}{2}, \frac{5}{2}\right]}+\mathcal{A}_{\left[10, \frac{5}{2}, \frac{1}{2}\right]}+\mathcal{A}_{\left[10, \frac{5}{2}, \frac{3}{2}\right]} \\
& +\mathcal{A}_{\left[10, \frac{3}{2}, \frac{5}{2}\right]}+2 \mathcal{A}_{\left[10, \frac{5}{2}, \frac{5}{2}\right]}+\ldots
\end{aligned}
$$

\section{B Generating function of characters}

To count the primaries in the $\mathrm{O}(N)$ vector model, we needed explicit expressions for the characters of $V_{+}^{\otimes n}$ projected to the trivial of $S_{n}\left[S_{2}\right]$. In this appendix we will derive the generating function

$$
\mathcal{Z}(t, Q)=\sum_{n=0}^{\infty} t^{n} \chi_{\mathcal{H}_{n}}(Q)
$$

The generating function of characters for $\mathcal{H}_{n}$, the $S_{n}\left[S_{2}\right]$ invariant subspace of $V^{\otimes 2 n}$, is

$$
\begin{aligned}
\mathcal{Z}(t, Q) & =\sum_{n=0}^{\infty} \frac{t^{n}}{2^{n} n !} \sum_{\sigma \in S_{n}\left[S_{2}\right]} \operatorname{tr}_{V} \otimes 2 n \\
& =\sum_{n=0}^{\infty} t^{n} \sum_{p \vdash 2 n} \mathcal{Z}_{\vec{p}}^{S_{n}\left[S_{2}\right]} \prod_{i}\left(\sum_{a} q_{a}^{i}\right)^{p_{i}} \sum_{n=0}^{\infty} \sum_{p \vdash 2 n} \mathcal{Z}_{\vec{p}}^{S_{n}\left[S_{2}\right]} \prod_{i}\left(\operatorname{tr} Q^{i}\right)^{p_{i}}
\end{aligned}
$$

Here $\mathcal{Z}_{\vec{p}}^{S_{n}\left[S_{2}\right]}$ is the number of permutations in $S_{n}\left[S_{2}\right]$ with cycle structure $\vec{p}$, divided by the order of $S_{n}\left[S_{2}\right]$. The cycle polynomials are

$$
\mathcal{Z}^{S_{n}\left[S_{2}\right]}(\vec{x})=\sum_{p \vdash 2 n} \mathcal{Z}_{\vec{p}}^{S_{n}\left[S_{2}\right]} \prod_{i} x_{i}^{p_{i}}
$$


The generating function of the cycle polynomials is given by

$$
\mathcal{Z}(t, \vec{x})=\sum_{n=0}^{\infty} t^{n} \mathcal{Z}^{S_{n}\left[S_{2}\right]}(\vec{x})=e^{\sum_{i=1}^{\infty} \frac{t^{i}}{2 i}\left(x_{2 i}+x_{i}^{2}\right)}
$$

Comparing (B.2) and (B.4) we see that

$$
\begin{aligned}
& \mathcal{Z}(t, Q)=\mathcal{Z}\left(t, x_{i} \rightarrow \sum_{a} q_{a}^{i}\right) \\
& =e^{\left.\sum_{i=1}^{\infty} \frac{t^{i}}{2 i}\left(\sum_{a} q_{a}^{i}\right)^{2}+\sum_{a} q_{a}^{2 i}\right)} \\
& =e^{\sum_{i=1}^{\infty} \frac{t^{i}}{2 i}\left(\sum_{a} \sum_{b} q_{a}^{i} q_{b}^{i}+\sum_{a} q_{a}^{2 i}\right)} \\
& =e^{\sum_{a, b} \sum_{i=1}^{\infty} \frac{t^{i} q_{a}^{i} q_{b}^{i}}{2 i}+\sum_{a} \sum_{i} \frac{t^{i}}{2 i} q_{a}^{2 i}} \\
& =e^{-\frac{1}{2} \sum_{a, b} \log \left(1-t q_{a} q_{b}\right)-\frac{1}{2} \sum_{a} \log \left(1-t q_{a}^{2}\right)} \\
& =\prod_{a} \frac{1}{\sqrt{1-q_{a}^{2}}} \prod_{a, b} \frac{1}{\sqrt{\left(1-t q_{a} q_{b}\right)}} \\
& =\prod_{a} \frac{1}{\sqrt{1-q_{a}^{2}}} \prod_{a<b} \frac{1}{\left(1-t q_{a} q_{b}\right)}
\end{aligned}
$$

\section{The Hilbert series for $Z_{3}(s, x, y)$}

In this appendix we consider the Hilbert series $Z_{3}(s, x, y)$ for the counting of extremal primaries built using 3 scalar fields. This Hilbert series has a non-trivial numerator

$$
Z_{3}^{z, w}=\frac{s^{3}\left(1-s^{5} x^{\frac{5}{2}}\left(\sqrt{y}+\frac{1}{\sqrt{y}}\right)-s^{6} x^{3}\left(\frac{1}{y}+1+y\right)-s^{14} x^{7}+s^{8} x^{4}\left(y+1+\frac{1}{y}\right)+s^{9} x^{\frac{9}{2}}\left(\sqrt{y}+\frac{1}{\sqrt{y}}\right)\right)}{\left(1-s^{2} x y\right)\left(1-s^{2} x\right)\left(1-s^{2} \frac{x}{y}\right)\left(1-s^{3} x^{\frac{3}{2}} y^{\frac{3}{2}}\right)\left(1-s^{3} x^{\frac{3}{2}} \sqrt{y}\right)\left(1-s^{3} \frac{x^{\frac{3}{2}}}{\sqrt{y}}\right)\left(1-\frac{s^{3} x^{\frac{3}{2}}}{y^{\frac{3}{2}}}\right)}
$$

Our goal in this appendix is to explain how the numerator of $Z_{3}(s, x, y)$ encodes relations between the generators of the ring as well as relations between those relations.

From the denominator of the Hilbert series, we have 7 generators. We can easily identify them as follows

$$
\begin{aligned}
G_{1}= & \left(z_{12}\right)^{2}+\left(z_{13}\right)^{2}+\left(z_{23}\right)^{2} & \leftrightarrow s^{2} x y \\
G_{2}= & z_{12} w_{12}+z_{13} w_{13}+z_{23} w_{23} & \leftrightarrow s^{2} x \\
G_{3}= & \left(w_{12}\right)^{2}+\left(w_{13}\right)^{2}+\left(w_{23}\right)^{2} & \leftrightarrow s^{2} \frac{x}{y} \\
G_{4}= & \left(z_{13}+z_{23}\right)\left(z_{31}+z_{21}\right)\left(z_{12}+z_{32}\right) & \leftrightarrow s^{3} x^{\frac{3}{2}} y^{\frac{3}{2}} \\
G_{5}= & \left(w_{13}+w_{23}\right)\left(z_{31}+z_{21}\right)\left(z_{12}+z_{32}\right) & \\
& +\left(z_{13}+z_{23}\right)\left(w_{31}+w_{21}\right)\left(z_{12}+z_{32}\right) & \\
& +\left(z_{13}+z_{23}\right)\left(z_{31}+z_{21}\right)\left(w_{12}+w_{32}\right) & \leftrightarrow s^{3} x^{\frac{3}{2}} y^{\frac{1}{2}} \\
G_{6}= & \left(w_{13}+w_{23}\right)\left(w_{31}+w_{21}\right)\left(z_{12}+z_{32}\right) & \\
& +\left(z_{13}+z_{23}\right)\left(w_{31}+w_{21}\right)\left(w_{12}+w_{32}\right) & \\
& +\left(w_{13}+w_{23}\right)\left(z_{31}+z_{21}\right)\left(w_{12}+w_{32}\right) & \leftrightarrow s^{3} x^{\frac{3}{2}} y^{-\frac{1}{2}} \\
G_{7}= & \left(w_{13}+w_{23}\right)\left(w_{31}+w_{21}\right)\left(w_{12}+w_{32}\right) & \leftrightarrow s^{3} x^{\frac{3}{2}} y^{-\frac{3}{2}}
\end{aligned}
$$


From the numerator of the Hilbert series, the terms with a negative sign should correspond to relations between the generators of the degree given by the monomial. From $-s^{5} x^{\frac{5}{2}}(\sqrt{y}+$ $\left.\frac{1}{\sqrt{y}}\right)-s^{6} x^{3}\left(\frac{1}{y}+1+y\right)-s^{14} x^{7}$ we have 6 relations. They are

$$
\begin{array}{ll}
\chi_{1}=3 G_{3} G_{4}-2 G_{2} G_{5}+G_{1} G_{6}=0 & \leftrightarrow s^{5} x^{\frac{5}{2}} \sqrt{y} \\
\chi_{2}=G_{3} G_{5}-2 G_{2} G_{6}+3 G_{1} G_{7}=0 & \leftrightarrow \frac{s^{5} x^{\frac{5}{2}}}{\sqrt{y}} \\
\chi_{3}=4 G_{1} G_{2}^{2}-G_{1}^{2} G_{3}=0 & \leftrightarrow s^{6} x^{3} y \\
\chi_{4}=4 G_{2}^{3}-G_{1} G_{2} G_{3}=0 & \leftrightarrow s^{6} x^{3} \\
\chi_{5}=4 G_{2}^{2} G_{3}-G_{1} G_{3}^{2}=0 & \leftrightarrow \frac{s^{6} x^{3}}{y} \\
\chi_{6}=G_{2}^{7}-G_{1} G_{2}^{5} G_{3}+\frac{1}{9} G_{2}^{4} G_{5} G_{6}-G_{2}^{4} G_{4} G_{7}=0 \leftrightarrow s^{14} x^{7}
\end{array}
$$

Again from the numerator of the Hilbert series, the terms with a positive sign should corresponds to relations between the relations, again of the degree given by the monomial. From $s^{8} x^{4}\left(y+1+\frac{1}{y}\right)+s^{9} x^{\frac{9}{2}}\left(\sqrt{y}+\frac{1}{\sqrt{y}}\right)$ we have 5 relations among the relations. They are

$$
\begin{aligned}
4 \chi_{5} G_{2}+\chi_{4} G_{3} & =0 \leftrightarrow \frac{s^{8} x^{4}}{y} \\
\chi_{5} G_{1}-\chi_{3} G_{3} & =0 \leftrightarrow s^{8} x^{4} \\
\chi_{4} G_{1}+4 \chi_{3} G_{2} & =0 \leftrightarrow s^{8} x^{4} y \\
\chi_{2} G_{2}^{2}-\frac{1}{4} \chi_{2} G_{1} G_{3}-\chi_{5} G_{5}-\frac{1}{2} \chi_{4} G_{6}-3 \chi_{3} G_{7} & =0 \leftrightarrow \frac{s^{9} x^{\frac{9}{2}}}{\sqrt{y}} \\
-4 \chi_{1} G_{2}^{2}+\chi_{1} G_{1} G_{3}+12 \chi_{5} G_{4}+2 \chi_{4} G_{5}+4 \chi_{3} G_{6} & =0 \leftrightarrow s^{9} x^{\frac{9}{2}} \sqrt{y}
\end{aligned}
$$

Open Access. This article is distributed under the terms of the Creative Commons Attribution License (CC-BY 4.0), which permits any use, distribution and reproduction in any medium, provided the original author(s) and source are credited.

\section{References}

[1] R. de Mello Koch and S. Ramgoolam, $C F T_{4}$ as $\mathrm{SO}(4,2)$-invariant $T F T_{2}$, Nucl. Phys. B 890 (2014) 302 [arXiv: 1403.6646] [INSPIRE].

[2] R. de Mello Koch and S. Ramgoolam, Strings from Feynman graph counting: without large-N, Phys. Rev. D 85 (2012) 026007 [arXiv:1110.4858] [InSPIRE].

[3] J. Pasukonis and S. Ramgoolam, Quivers as calculators: counting, correlators and Riemann surfaces, JHEP 04 (2013) 094 [arXiv:1301.1980] [INSPIRE].

[4] R. de Mello Koch and S. Ramgoolam, From matrix models and quantum fields to Hurwitz space and the absolute Galois group, arXiv:1002.1634 [INSPIRE].

[5] J. Ben Geloun and S. Ramgoolam, Counting tensor model observables and branched covers of the 2-sphere, arXiv:1307.6490 [INSPIRE]. 
[6] S. Rychkov and Z.M. Tan, The $\epsilon$-expansion from conformal field theory, J. Phys. A 48 (2015) 29FT01 [arXiv:1505.00963] [INSPIRE].

[7] P. Basu and C. Krishnan, $\epsilon$-expansions near three dimensions from conformal field theory, JHEP 11 (2015) 040 [arXiv: 1506.06616] [INSPIRE].

[8] S. Ghosh, R.K. Gupta, K. Jaswin and A.A. Nizami, $\epsilon$-expansion in the Gross-Neveu model from conformal field theory, JHEP 03 (2016) 174 [arXiv:1510.04887] [INSPIRE].

[9] A. Raju, $\epsilon$-expansion in the Gross-Neveu CFT, JHEP 10 (2016) 097 [arXiv:1510.05287] [INSPIRE].

[10] K. Nii, Classical equation of motion and anomalous dimensions at leading order, JHEP 07 (2016) 107 [arXiv : 1605. 08868] [INSPIRE].

[11] F. Gliozzi, A. Guerrieri, A.C. Petkou and C. Wen, Generalized Wilson-Fisher critical points from the conformal operator product expansion, Phys. Rev. Lett. 118 (2017) 061601 [arXiv: 1611.10344] [INSPIRE].

[12] F. Gliozzi, A.L. Guerrieri, A.C. Petkou and C. Wen, The analytic structure of conformal blocks and the generalized Wilson-Fisher fixed points, JHEP 04 (2017) 056 [arXiv: 1702.03938] [INSPIRE].

[13] F.A. Dolan, Character formulae and partition functions in higher dimensional conformal field theory, J. Math. Phys. 47 (2006) 062303 [hep-th/0508031] [INSPIRE].

[14] T.H. Newton and M. Spradlin, Quite a character: the spectrum of Yang-Mills on $S^{3}$, Phys. Lett. B 672 (2009) 382 [arXiv:0812.4693] [INSPIRE].

[15] T.W. Brown, P.J. Heslop and S. Ramgoolam, Diagonal free field matrix correlators, global symmetries and giant gravitons, JHEP 04 (2009) 089 [arXiv:0806.1911] [INSPIRE].

[16] I. Frenkel and M. Libine Quarternionic analysis, representation theory and physics, Adv. Math. 218 (2008) 1806.

[17] J. Gray, A. Hanany, Y.-H. He, V. Jejjala and N. Mekareeya, SQCD: a geometric apercu, JHEP 05 (2008) 099 [arXiv: 0803.4257] [INSPIRE].

[18] A. Hanany, N. Mekareeya and G. Torri, The Hilbert series of adjoint SQCD, Nucl. Phys. B 825 (2010) 52 [arXiv:0812.2315] [INSPIRE].

[19] R. Stanley, Hilbert functions of graded algebras, Adv. Math. 28 (1978) 57.

[20] M.R. Gaberdiel and R. Gopakumar, Higher spins \& strings, JHEP 11 (2014) 044 [arXiv: 1406.6103] [INSPIRE].

[21] R. de Mello Koch, P. Rabambi, R. Rabe and S. Ramgoolam, Free quantum fields in $4 D$ and Calabi-Yau spaces, to appear.

[22] V.K. Dobrev, V.B. Petkova, S.G. Petrova and I.T. Todorov, Dynamical derivation of vacuum operator product expansion in euclidean conformal quantum field theory, Phys. Rev. D 13 (1976) 887 [INSPIRE].

[23] M.S. Costa, J. Penedones, D. Poland and S. Rychkov, Spinning conformal correlators, JHEP 11 (2011) 071 [arXiv:1107.3554] [INSPIRE].

[24] R. Goodman and N.R. Wallach, Representations and invariants of the classical groups, Cambridge University Press, Cambridge U.K. (1998). 
[25] S. Minwalla, Restrictions imposed by superconformal invariance on quantum field theories, Adv. Theor. Math. Phys. 2 (1998) 781 [hep-th/9712074] [INSPIRE].

[26] K. Roumpedakis, Leading order anomalous dimensions at the Wilson-Fisher fixed point from CFT, JHEP 07 (2017) 109 [arXiv:1612.08115] [INSPIRE].

[27] M. Hamermesh, Group theory and its application to physical problems, Addison-Wesley Publishing Company Inc., U.S.A. (1962).

[28] S. Giombi and V. Kirilin, Anomalous dimensions in CFT with weakly broken higher spin symmetry, JHEP 11 (2016) 068 [arXiv:1601.01310] [INSPIRE].

[29] M. Hochster and J. Roberts, Rings of invariants of reductive groups acting on regular rings are Cohen-Macaulay, Adv. Math. 13 (1974) 115.

[30] I.R. Klebanov and A.M. Polyakov, AdS dual of the critical $\mathrm{O}(N)$ vector model, Phys. Lett. B 550 (2002) 213 [hep-th/0210114] [INSPIRE].

[31] M.A. Vasiliev, Consistent equation for interacting gauge fields of all spins in (3+1)-dimensions, Phys. Lett. B 243 (1990) 378 [INSPIRE].

[32] F.A. Dolan, Counting BPS operators in N=4 SYM, Nucl. Phys. B 790 (2008) 432 [arXiv:0704.1038] [INSPIRE].

[33] R. de Mello Koch, P. Diaz and H. Soltanpanahi, Non-planar anomalous dimensions in the sl(2) sector, Phys. Lett. B 713 (2012) 509 [arXiv:1111.6385] [InSPIRE].

[34] O. Aharony, J. Marsano, S. Minwalla, K. Papadodimas and M. Van Raamsdonk, The Hagedorn-deconfinement phase transition in weakly coupled large- $N$ gauge theories, Adv. Theor. Math. Phys. 8 (2004) 603 [hep-th/0310285] [INSPIRE].

[35] P.J. Cameron, Combinatorics: topics, techniques, algorithms, Cambridge University Press, Cambridge U.K. (1994).

[36] M.A. Vasiliev, Multiparticle extension of the higher-spin algebra, Class. Quant. Grav. 30 (2013) 104006 [arXiv:1212.6071] [INSPIRE].

[37] O.A. Gelfond and M.A. Vasiliev, Operator algebra of free conformal currents via twistors, Nucl. Phys. B 876 (2013) 871 [arXiv:1301.3123] [INSPIRE].

[38] G. Benkart, T. Halverson and N. Harman, Dimensions of irreducible modules for partition algebras and tensor power multiplicities for symmetric and alternating groups, arXiv: 1605.06543.

[39] V.M. Braun, G.P. Korchemsky and D. Mueller, The uses of conformal symmetry in QCD, Prog. Part. Nucl. Phys. 51 (2003) 311 [hep-ph/0306057] [INSPIRE].

[40] S. Corley, A. Jevicki and S. Ramgoolam, Exact correlators of giant gravitons from dual $N=4$ SYM theory, Adv. Theor. Math. Phys. 5 (2002) 809 [hep-th/0111222] [INSPIRE].

[41] D. Berenstein, A Toy model for the AdS/CFT correspondence, JHEP 07 (2004) 018 [hep-th/0403110] [INSPIRE].

[42] H. Lin, O. Lunin and J.M. Maldacena, Bubbling AdS space and 1/2 BPS geometries, JHEP 10 (2004) 025 [hep-th/0409174] [INSPIRE].

[43] L. Grant, P.A. Grassi, S. Kim and S. Minwalla, Comments on 1/16 BPS quantum states and classical configurations, JHEP 05 (2008) 049 [arXiv: 0803.4183] [INSPIRE]. 
[44] J. Pasukonis and S. Ramgoolam, From counting to construction of BPS states in $N=4$ SYM, JHEP 02 (2011) 078 [arXiv: 1010.1683] [INSPIRE].

[45] J. Pasukonis and S. Ramgoolam, Quantum states to brane geometries via fuzzy moduli spaces of giant gravitons, JHEP 04 (2012) 077 [arXiv: 1201.5588] [INSPIRE].

[46] A. Mikhailov, Giant gravitons from holomorphic surfaces, JHEP 11 (2000) 027 [hep-th/0010206] [INSPIRE].

[47] I. Biswas, D. Gaiotto, S. Lahiri and S. Minwalla, Supersymmetric states of $N=4$ Yang-Mills from giant gravitons, JHEP 12 (2007) 006 [hep-th/0606087] [INSPIRE].

[48] R. de Mello Koch, M. Dessein, D. Giataganas and C. Mathwin, Giant graviton oscillators, JHEP 10 (2011) 009 [arXiv:1108.2761] [INSPIRE].

[49] R. de Mello Koch and S. Ramgoolam, A double coset ansatz for integrability in AdS/CFT, JHEP 06 (2012) 083 [arXiv: 1204.2153] [INSPIRE].

[50] D. Berenstein, Giant gravitons: a collective coordinate approach, Phys. Rev. D 87 (2013) 126009 [arXiv: 1301.3519] [INSPIRE].

[51] D. Berenstein, Sketches of emergent geometry in the gauge/gravity duality, Fortsch. Phys. 62 (2014) 776 [arXiv:1404.7052] [INSPIRE].

[52] Y. Kimura and S. Ramgoolam, Branes, anti-branes and brauer algebras in gauge-gravity duality, JHEP 11 (2007) 078 [arXiv:0709.2158] [INSPIRE].

[53] T.W. Brown, P.J. Heslop and S. Ramgoolam, Diagonal multi-matrix correlators and BPS operators in $N=4$ SYM, JHEP 02 (2008) 030 [arXiv:0711.0176] [INSPIRE].

[54] R. Bhattacharyya, S. Collins and R. de Mello Koch, Exact multi-matrix correlators, JHEP 03 (2008) 044 [arXiv: 0801.2061 [ [nSPIRE].

[55] R. Bhattacharyya, R. de Mello Koch and M. Stephanou, Exact multi-restricted schur polynomial correlators, JHEP 06 (2008) 101 [arXiv:0805.3025] [INSPIRE].

[56] J. Pasukonis and S. Ramgoolam, Quivers as calculators: counting, correlators and Riemann surfaces, JHEP 04 (2013) 094 [arXiv:1301.1980] [INSPIRE].

[57] R. de Mello Koch, R. Kreyfelt and N. Nokwara, Finite $N$ quiver gauge theory, Phys. Rev. D 89 (2014) 126004 [arXiv: 1403.7592] [INSPIRE]. 